\title{
Multi-model assessment of the late-winter stratospheric response to EI Niño and La Niña
}

\author{
Bianca Mezzina ${ }^{1}$. Froila M. Palmeiro² . Javier García-Serrano ${ }^{1,2}$ - Ileana Bladé2 Lauriane Batté $^{3}$. \\ Marianna Benassi ${ }^{4}$
}

Received: 10 December 2020 / Accepted: 3 June 2021 / Published online: 13 June 2021

(c) The Author(s) 2021

\begin{abstract}
The impact of El Niño-Southern Oscillation (ENSO) on the late-winter extra-tropical stratosphere (January-March) is assessed in a multi-model framework. Three state-of-the-art atmospheric models are run with prescribed SST anomalies representative of a strong ENSO event, with symmetric patterns for El Niño and La Niña. The well-known temperature perturbation in the lower stratosphere during El Niño is captured by two models, in which the anomalous warming at polar latitudes is accompanied by a positive geopotential height anomaly that extends over the polar cap. In the third model, which shows a lack of temperature anomalies over the pole, the anomalous anticyclone is confined over Canada and does not expand to the polar cap. This anomalous center of action emerges from the large-scale tropospheric Rossby wave train forced by ENSO, and shrinking/stretching around the polar vortex is invoked to link it to the temperature response. No disagreement across models is found in the lower stratosphere for La Niña, whose teleconnection is opposite in sign but weaker. In the middle-upper stratosphere (above $50 \mathrm{hPa}$ ) the geopotential height anomalies project on a wavenumber-1 (WN1) pattern for both El Niño and, more weakly, La Niña, and show a westward tilt with height up to the stratopause. It is suggested that this WN1 pattern arises from the high-latitude lower-stratospheric anomalies, and that the ENSO teleconnection to the polar stratosphere can be interpreted in terms of upward propagation of the stationary Rossby wave train and quasi-geostrophic balance, instead of wave breaking.
\end{abstract}

Keywords ENSO $\cdot$ Atmospheric teleconnections $\cdot$ Climate modeling $\cdot$ Stratosphere

\section{Introduction}

This paper is a contribution tothe MEDSCOPE special issue on the drivers of variability andsources of predictability for the European and Mediterranean regionsat subseasonal to multiannual time scales. MEDSCOPE is anERA4CS project co-funded by JPI Climate. The special issue wascoordinated by Silvio Gualdi and Lauriane Batté.

Bianca Mezzina

bianca.mezzina@bsc.es

Barcelona Supercomputing Center (BSC), Barcelona, Spain

2 Group of Meteorology, Universitat de Barcelona (UB), Barcelona, Spain

3 CNRM, Université de Toulouse, Météo-France, CNRS, Toulouse, France

4 Fondazione Centro Euro-Mediterraneo sui Cambiamenti Climatici (CMCC), Bologna, Italy
When referring to El Niño-Southern Oscillation (ENSO), the term "stratospheric pathway" is now commonly used to allude to its extra-tropical teleconnection (e.g. Butler et al. 2014). With this expression, it is implied that not only is ENSO inducing a response in the stratosphere, but also that this response is later transferred to the surface. Concerning the first part of the pathway, namely how the stratosphere is affected by ENSO, El Niño has been shown to have a robust impact on the winter seasonal-mean state of the Northern Hemisphere polar stratosphere, consisting of a warming at low levels and a weakening of the westerly flow related to the polar vortex (e.g. Taguchi and Hartmann 2006; Free and Seidel 2009; Calvo et al. 2010; see Domeisen et al. 2019 for a review). More recently, La Niña has been associated with the opposite response, a lower-stratospheric cooling and a strengthening of the westerly flow linked to the polar vortex (e.g. Calvo et al. 2010; Hurwitz et al. 2014; Iza et al. 
2016; Weinberger et al. 2019), although there is still some controversy as to whether this atmospheric response is truly symmetric (e.g. Manzini et al. 2006; Hurwitz et al. 2014; Rao and Ren 2016a, b; Hardiman et al. 2019), where the skewness of the ENSO forcing may play a role. Addressing the (a)symmetry of the ENSO stratospheric response is one of the aims of the present model-based study, taking advantage of an experimental set-up with symmetric idealized SST forcing to represent El Niño and La Niña.

The mechanisms leading to the ENSO response in the polar stratosphere are also unsettled. The well-known tropospheric Rossby wave train excited by ENSO (Horel and Wallace 1981; Hoskins and Karoly 1981) has its first center of action over the North Pacific and a second one over Canada. Early studies suggested that during El Niño the stationary wave pattern is reinforced in the lower stratosphere by the center of action over Canada, which strengthens the climatological Aleutian High and vertically propagates to the mesosphere (e.g. van Loon and Labitzke 1987; Hamilton 1993a, b, 1995; Sassi et al. 2004; Manzini et al. 2006). In contrast, a more recent interpretation of the ENSO impact on the polar stratosphere relies on the first center of action, which strengthens and expands (weakens and contracts) the climatological Aleutian Low in the troposphere during El Niño (La Niña). Thus, for El Niño, linear constructive interference is suggested to take place between the perturbed Aleutian Low and the climatological stationary wave pattern, and the opposite for La Niña (e.g. Garfinkel and Hartmann 2008; Ineson and Scaife 2009). According to this view, during El Niño anomalous vertically-propagating planetary waves grow with height and eventually break, decelerating the polar vortex and warming the stratosphere (e.g. see Domeisen et al. 2019 for a review), while wave breaking inhibition is associated with a stronger vortex during $\mathrm{La}$ Niña (e.g. Iza et al. 2016). In this study, we examine the vertical structure of the ENSO-forced circulation anomalies and their interaction with the climatological state and provide evidence to support the key roles of the center of action over Canada and the stratospheric Aleutian High, as opposed to the tropospheric Aleutian Low. In addition, by combining this analysis with other diagnostics such as the EliassenPalm (EP) flux and its divergence, we revisit the paradigm of the stratospheric impact of ENSO through anomalous "irreversible" (see Waugh and Polvani 2010 for a review) wave breaking, with arguments based on large-scale dynamics, including quasi-geostrophy and thermal wind balance.

Historically, the hypothesis of the stratospheric pathway emerged in an effort to understand the elusive ENSO teleconnection to the North Atlantic-European (NAE) region (e.g. Brönnimann 2007). The idea is that, as suggested for other stratospheric perturbations (see Kidston et al. 2015 for a review), the stratospheric ENSO signal may propagate downwards and project onto a dipolar sea-level pressure
(SLP) pattern over the North Atlantic. This would be in agreement with the observed late-winter (January-March) "canonical" response to ENSO in the NAE sector, which consists of a SLP dipole between mid and high latitudes (see Brönnimann 2007 for a review). Mezzina et al. (2021) have used the same multi-model ensemble employed in the present study to analyze the tropospheric pathway of the ENSO teleconnection and have concluded that this dipole (Fig. 1) is mostly driven by the forced, tropospheric large-scale Rossby wave train. By assessing the stratospheric response in the same set of sensitivity experiments, we complement their results and provide further evidence for the minor role of the stratosphere in the late-winter ENSO teleconnection to the NAE region.

Sudden stratospheric warmings (SSWs) are abrupt, nonlinear events that consist of a strong deceleration and warming of the polar vortex (Matsuno 1971), similar in sign to the El Niño effect, but with a different time-scale (dailyweekly). They have been suggested to play a key role in the stratospheric pathway of El Niño to the NAE region (e.g. Domeisen et al. 2015; Richter et al. 2015; Calvo et al. 2017; Bell et al. 2009; Ineson and Scaife 2009; Butler et al. 2014), or an amplifying role for the surface response (Cagnazzo and Manzini 2009). The relationship with the La Niña teleconnection is more obscure, and overall their implication during ENSO events is not clear, as other studies consider SSWs and ENSO as distinct sources of variability in the North Atlantic (e.g. Polvani et al. 2017; Oehrlein et al. 2019). Using the same set of sensitivity experiments as in the present study, Palmeiro et al. (2021a) have concluded that while ENSO modifies the seasonal-mean state of the polar vortex by conditioning it to be more (less) easily perturbed during El Niño (La Niña), the actual triggering of SSWs (at higher-frequency time-scales) is largely unrelated to ENSO. Based on their results, in this work only anomalies at the seasonal time-scale are considered, which implicitly include SSWs, but the occurrence of SSWs is not explicitly taken into account, also in agreement with their minimal contribution to the seasonal-mean state (Garfinkel et al. 2012).

This study explores the impact of ENSO on the polar stratosphere in late winter (JFM; January-March), following the approach of Mezzina et al. $(2020,2021)$ to avoid intra-seasonal issues between early and late winter in the canonical NAE response (e.g. Ayarzagüena et al. 2018; King et al. 2018), and also in recognition of the fact that the stratospheric response to El Niño is not well established until January (e.g. van Loon and Labitzke 1987; Manzini et al. 2006; Ineson and Scaife 2009). Several model-based studies have been published on this topic, but the fact that the same suite of sensitivity experiments is run using three state-of-the-art high-top models (i.e., with a well-resolved stratosphere) adds strength to this work. Furthermore, the experimental design, tailored to isolate the impacts of a 
(a) EN (EC-EARTH) Z850

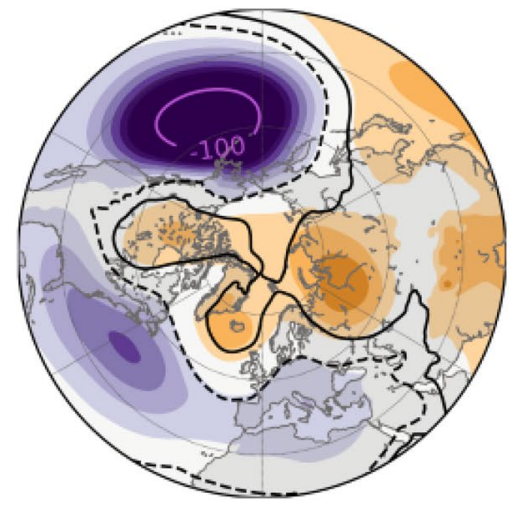

(d) LN (ECEARTH) Z850

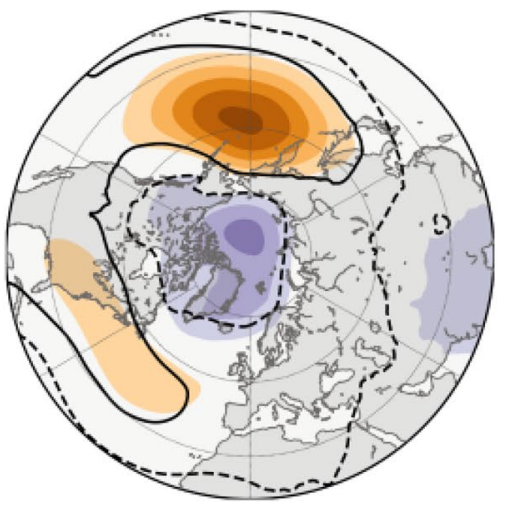

(b) EN (CNRM) Z850

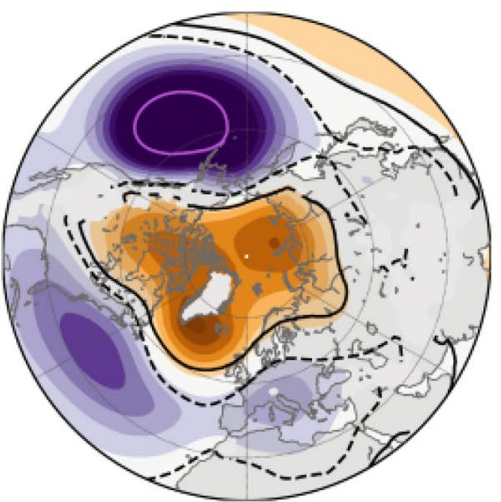

(e) LN (CNRM) Z850

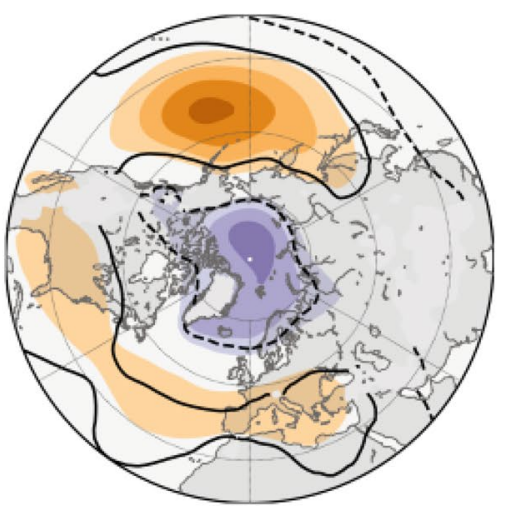

(c) EN (CMCC) Z850

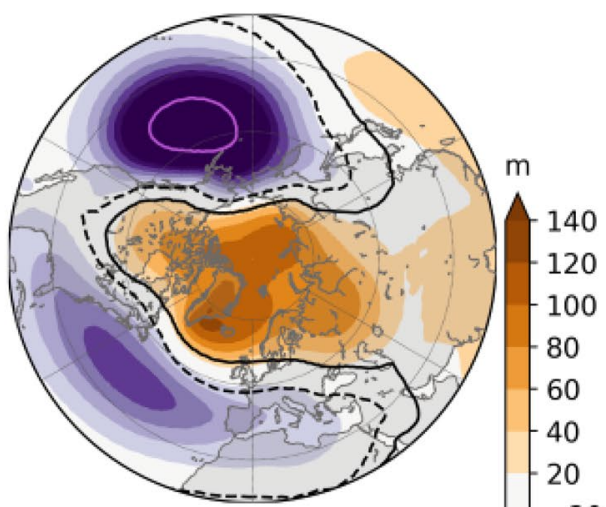

(f) LN (CMCC) Z850

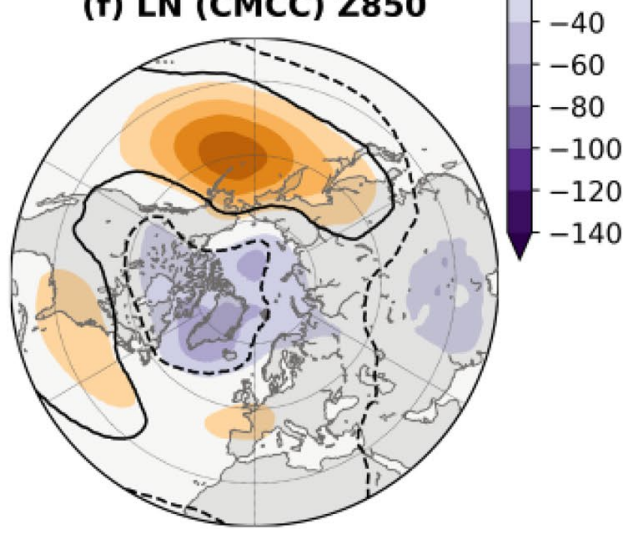

Fig. 1 Ensemble-mean geopotential height response at $850 \mathrm{hPa}$ for EN (top) and LN (bottom) in JFM: EC-EARTH (left), CNRM (middle), CMCC (right). Magenta contours show values exceeding

symmetric, observation-based El Niño- and La Niña-like forcing, distinguishes our study from previous ones using coupled models (e.g. Calvo et al. 2017), global SST forcing (e.g. Weinberger et al. 2019) or idealized boundary conditions (e.g. Trascasa-Castro et al. 2019).

We will assess, in a multi-model approach, the (a)symmetry of the late-winter response to El Niño and La Niña in the lower (up to $50 \mathrm{hPa}$ ) and middle-upper (up to $1 \mathrm{hPa}$ ) stratosphere separately, to highlight the different features. Taking advantage of the different performances of the models, we will clarify what aspects of the ENSO response in the polar stratosphere appear to be robust and we will provide a consistent dynamical interpretation and/or implications for the impact on temperature and zonal wind.

After describing the experiments and methodology (Sect. 2), the models' climatology in the stratosphere is briefly analyzed (Sect. 3.1). Results on the SST-forced atmospheric circulation are presented in the rest of Sect. 3, first examining the zonal-mean response (Sects. 3.2-3.3), then focusing on the lower stratosphere (Sects. 3.4-3.5) and finally on the middle-upper stratosphere (Sect. 3.6). Additional remarks on the dynamical aspects of the temperature the color scale limit at $-100 \mathrm{~m}$. Black contours (solid for positive, dashed for negative anomalies) indicate statistically significant areas at the $95 \%$ confidence level

response are presented in Sect. 3.7. The main findings are summarized and discussed in Sect. 4.

\section{Data and methods}

\subsection{Models and experimental set-up}

This study makes use of a coordinated set of sensitivity atmosphere-only experiments performed with three different state-of-the-art atmospheric general circulation models (AGCMs) that contribute to the European ERA4CS-funded MEDSCOPE project. The experimental protocol, aimed to isolate the atmospheric response to El Niño (EN) and La Niña (LN), as compared to neutral conditions with prescribed climatological SST (CTL), has already been described in depth by Mezzina et al. (2021) and Benassi et al. (2021). Here, only a short summary is provided. Common to all simulations is: (i) the background SST climatology, computed from HadISST v2.2 (Titchner and Rayner 2014) over 1981-2010; (ii) fixed radiative forcings (GHG, solar, ozone, aerosols) at year 2000 , representative of 
present-day conditions; and, (iii) the ensemble size, consisting of 50 winters. The baseline experiment (CTL) was run for 50 years after spin-up, providing atmospheric initial conditions for the ENSO experiments. The anomalous boundary conditions were created by regressing detrended monthly SST anomalies onto the DJF Niño3.4 index to set an ENSO cycle from June (year 0) to May (year 1), restricted to the central-eastern tropical Pacific (e.g. as in Jiménez-Esteve and Domeisen 2019), and amplified to have a maximum anomaly of $2.7 / 2.4{ }^{\circ} \mathrm{C}$ in DJF/JFM (e.g. as in Taguchi and Hartmann 2006). The EN and LN SST forcings are symmetric and opposite in sign and can be considered as idealized strong eastern-Pacific events. Further details, including visualization of the SST patterns, can be found in Mezzina et al. (2021).

The AGCMs correspond to the atmospheric components of the following climate models (with lon $\times$ lat grid/vertical levels): EC-EARTH version 3.2, $512 \times 256 /$ L91 up to $0.01 \mathrm{hPa}$ (Davini et al. 2017); CNRM Climate Model version 6-1, 256×128/L91 up to $0.01 \mathrm{hPa}$ (Voldoire et al. 2019; Roehrig et al. 2020); CMCC Seasonal Prediction System $3,360 \times 180 / \mathrm{L} 46$ up to $0.3 \mathrm{hPa}$ (Sanna et al. 2017). These three AGCMs are considered "high-top" models since they properly resolve the stratosphere, particularly the northern polar stratosphere. They also simulate QBO-like variability in the tropical stratosphere (e.g. see Palmeiro et al. 2020 for EC-EARTH), but the potential impact of the (internally-generated) QBO on the ENSO teleconnections has been minimized as all simulations start from the same atmospheric initial conditions from a continuous run, so that the different easterly/westerly QBO phases are equally sampled in the CTL and EN/LN experiments. More details on the AGCMs can be found in Mezzina et al. (2021).

\subsection{Diagnostics and methods}

In quasi-geostrophic (QG) theory, large-scale Rossby wave propagation and its interaction with the mean flow can be diagnosed by the Eliassen-Palm (EP) flux (e.g. Andrews et al. 1987; Vallis 2017):

$F_{y}=-\rho a \cos \varphi\left[u^{*} v^{*}\right]$

$F_{z}=\rho a \cos \varphi \frac{R f}{H N^{2}}\left[v^{*} T^{*}\right]$

where $\rho$ is the air density, $a$ is the Earth radius, $\varphi$ is latitude, $R$ is the gas constant $\left(287 \mathrm{~m}^{2} \mathrm{~s}^{-2} \mathrm{~K}^{-1}\right), f$ is the Coriolis parameter or planetary vorticity, $H$ is the scale-height $(7 \mathrm{~km})$, and $N^{2}$ is the squared Brunt-Väisälä buoyancy frequency. $\left[u^{*} v^{*}\right]$ is the eddy momentum flux and $\left[v^{*} T^{*}\right]$ the eddy heat flux, where $*$ indicates perturbation from the zonal-mean and [] denotes a zonal-mean. The divergence of the EP flux $(\nabla \cdot F)$ appears in the momentum equation and encapsulates the eddy forcing of the mean flow, with EP flux divergence (convergence) implying acceleration (deceleration) of the zonal-mean zonal wind. These diagnostics are examined in Sect. 3.3. Details on the scaling of $F_{z}$ and $F_{y}$ are described in the caption of Fig. 4.

The QG potential vorticity can be defined as (e.g. Andrews et al. 1987; Vallis 2017):

$q=\zeta+f+\frac{1}{\rho} \frac{\partial}{\partial z}\left(\rho \frac{f_{0}^{2}}{N^{2}} \frac{\partial \psi}{\partial z}\right)$

where $g$ is the gravity, $f_{0}$ is a suitable reference of the Coriolis parameter at some origin $\varphi_{0}$ (note that $\Phi=f_{0} \psi$, with $\Phi$ the geopotential), $\psi$ is the geostrophic streamfunction, and $z$ is the vertical log-pressure coordinate. The first term is the geostrophic relative vorticity $\left(\zeta=\nabla^{2} \psi\right)$, the second represents the planetary vorticity $(f=2 \Omega \sin \varphi)$, and the third term is the stretching vorticity. The potential vorticity is conserved following the (geostrophic) flow in the absence of frictional and diabatic effects. The vertical gradient of $\psi$ (and its curvature, the second derivative) increases (decreases) where relative vorticity decreases or becomes more anticyclonic (increases or becomes more cyclonic). This can be more easily diagnosed by taking the (zonal-eddy) perturbation potential vorticity (e.g. Plumb 1985; Andrews et al. 1987):

$q^{*}=\zeta^{*}+\frac{1}{\rho} \frac{\partial}{\partial z}\left(\rho \frac{f_{0}^{2}}{N^{2}} \frac{\partial \psi^{*}}{\partial z}\right)$

Note that the two views presented above are dynamically equivalent. In fact, from the zonal-mean QG potential vorticity equation, the divergence of the EP flux can be expressed as (e.g. Andrews et al. 1987; Vallis 2017): $\rho^{-1} \nabla \cdot F=\left[v^{*} q^{*}\right]$; thereby, EP flux divergence (convergence) is associated with a poleward flux of positive (negative) potential vorticity.

Furthermore, in hydrostatic balance, changes in potential vorticity can be related to temperature variations via the hypsometric equation (e.g. Andrews et al. 1987):

$T^{*}=\frac{H f_{0}}{R} \frac{\partial \psi^{*}}{\partial z}$

The link between potential vorticity and temperature might be more easily interpreted using isentropic coordinates and Ertel's formulation (e.g. Vallis 2017), but this is beyond the scope of the present study.

Ensemble-mean differences between the EN/LN experiments and the CTL simulation are computed to estimate the forced atmospheric response to ENSO. Statistical significance has been assessed with a Student's $t$-test for 
difference of means at the $95 \%$ confidence level. Note that while monthly outputs were stored at several vertical levels from 850 to $1 \mathrm{hPa}$, for daily outputs only seven vertical levels in the stratosphere were available from the multimodel: 200, 100, 70, 50, 30, 20, $10 \mathrm{hPa}$. Observational composites based on reanalysis are shown and detailed in Appendix 1.

\section{Results}

\subsection{Stratosphere climatology}

Since the aim of the study is to investigate the impact of ENSO on the stratosphere, we first describe the models climatological geopotential height (Z) at various levels (Fig. 2). In the lower stratosphere (at 100 and $50 \mathrm{hPa}$, Fig. 2g-1), the low-pressure center corresponding to the polar vortex (shading) is slightly off-centered with respect to the pole and elongates towards Siberia and Canada, while it retracts over the North Pacific due to the presence of a large-scale anticyclone (the Rocky Mountain Ridge; Nigam and DeWeaver 2003) that appears clearly in the zonal-eddy field ( $Z^{*}$, contours). In the middle-upper stratosphere (at 10 and $1 \mathrm{hPa}$ ) the vortex is much more zonally symmetric and stronger (Fig. 2a-f, shading), although still showing marked deviations from the pole. Here, the zonal-eddy field is dominated by an anticyclone, the Aleutian High, and a cyclone, representing a displacement of the vortex: together, they form a couplet that tilts westward with height, indicating upward propagation of stationary wave activity, into the mesosphere (Harvey and Hitchman 1996; Harvey et al. 2002). With increasing height, the westerly winds strengthen so that only the longest waves can propagate upwards (Charney-Drazin wave filtering; Charney and Drazin 1961). Hence at 10 and $1 \mathrm{hPa}$ a predominantly wavenumber-1 (WN1) pattern is found in the $Z^{*}$ field (Fig. 2a-f), while some WN2 component is present at $50 \mathrm{hPa}$ (Fig. 2g-i) and additionally some WN3 component at $100 \mathrm{hPa}$ (Fig. 2j-1). All models capture these climatological features well, although EC-EARTH systematically shows a weaker vortex, particularly at 50 and $10 \mathrm{hPa}$ (Fig. 2, left column; see Fig. 3a, g), a known bias of this model (Palmeiro et al. 2021b).

\subsection{Zonal-mean zonal wind and temperature}

We begin the analysis of the ENSO impact on the stratosphere by examining the anomalous response of the zonalmean zonal wind in the EN experiment (Fig. 3, top row). A negative anomaly north of $60^{\circ} \mathrm{N}$ is present in CNRM and $\mathrm{CMCC}$, peaking in the middle stratosphere $(\sim 10 \mathrm{hPa})$ and extending from the lowermost stratosphere to beyond $1 \mathrm{hPa}$ (although the statistical significance is model dependent;
Fig. 3b,c), implying a deceleration of the polar vortex (Fig. 3b, c, green contours). In both models, this weakening of the westerly winds is accompanied by a warming of the lower stratosphere north of $70^{\circ} \mathrm{N}$ (Fig. 3e,f), peaking at approximately $100 \mathrm{hPa}$ and extending from just above the tropopause (green contour) to $\sim 10 \mathrm{hPa}$, consistent with thermal wind balance. Both the weakening of the vortex, in the middle-upper stratosphere, and the warming of the polar cap, in the lower stratosphere, are well-documented aspects of the El Niño teleconnection to the stratosphere and also appear in our observational composites (Fig. 11a, c in Appendix 1). A different response, however, is found in the third model, EC-EARTH, which fails to capture the expected changes in the stratospheric polar cap (Fig. 3a, d). Note that, in contrast, in the troposphere, a southward shift in the mid-latitude jet is observed in all models (Fig. 3, top).

Interestingly, EC-EARTH does not show the same wrong performance for $\mathrm{LN}$ : all three models show a robust increase in the zonal-mean zonal wind at subpolar latitudes maximizing at around $10 \mathrm{hPa}$, as well as a cooling over the lower stratospheric polar cap (Fig. 3, third and fourth rows), again consistent with thermal wind balance. In CMCC, the response in $\mathrm{EN}$ is about 50\% stronger than in $\mathrm{LN}$, which is also less significant (cf. Fig. 3c, f, i, and 1); on the contrary, in CNRM, the magnitude of the anomalies appears comparable or even stronger in LN (cf. Fig. 3b, e, h, and k), but, as we will discuss later in Sect. 3.6, this is a consequence of the zonal average. This almost linear response for $\mathrm{LN}$ was not necessarily expected - although also found in other models (e.g. Calvo et al. 2010; Hurwitz et al. 2014; Trascasa-Castro et al. 2019; Weinberger et al. 2019)—given that the observed La Niña signal is weak and not significant in our composites (Fig. 11e, g) or other observational studies (e.g. Manzini et al. 2006).

Note also that EC-EARTH simulates a weaker, southward shifted polar vortex compared to CNRM and CMCC (Fig. 3, green contours). This difference in the mean flow may have some impacts, but it is unlikely a relevant factor for the discordant EN signal in EC-EARTH, given that the same behavior is not observed for LN. This peculiarity of the ENSO response in EC-EARTH and the comparison with the other two models offers a unique opportunity to clarify the key mechanisms of the ENSO teleconnection to the stratosphere.

\subsection{Eliassen-Palm flux and divergence}

Changes in the upward propagation and breaking of planetary waves is commonly considered as the main factor driving the ENSO impact on the polar stratosphere. Failure in capturing this anomalous wave-mean flow interaction may be responsible for the unrealistic response in the zonal-mean zonal wind and temperature in EC-EARTH for 
(a) CTL (EC-EARTH) Z1

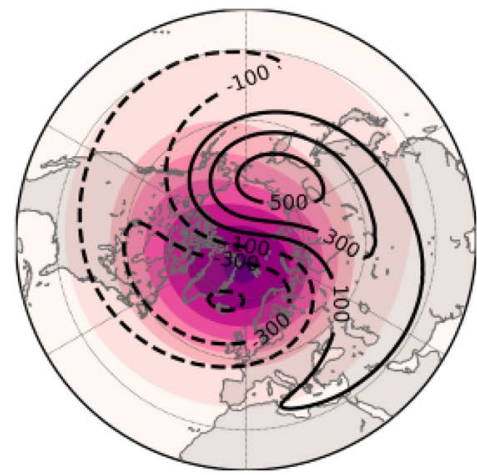

(d) CTL (EC-EARTH) Z10

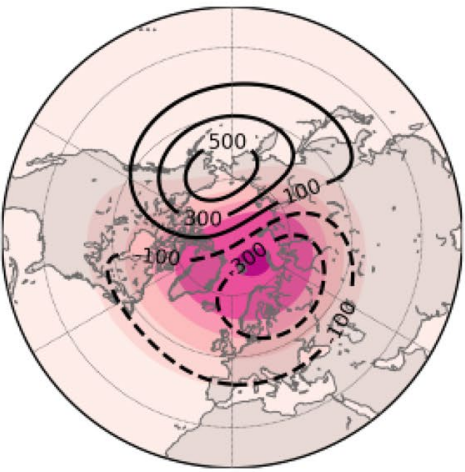

(g) CTL (EC-EARTH) Z50

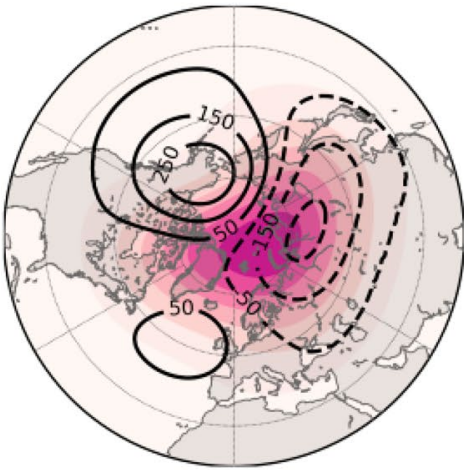

(j) CTL (EC-EARTH) Z100

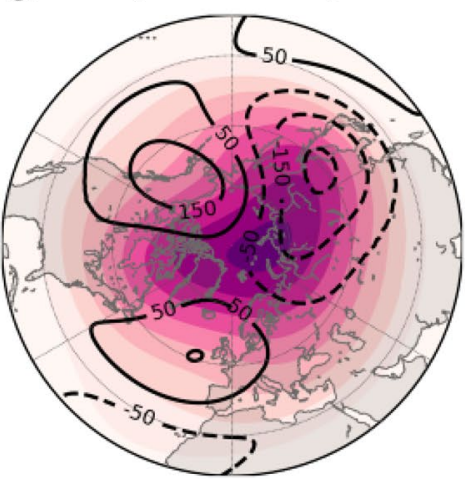

(b) CTL (CNRM) Z1

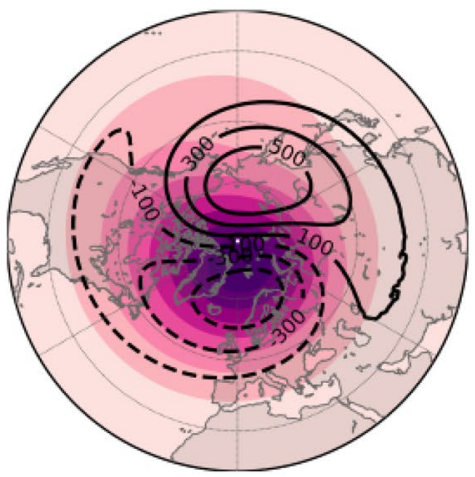

(e) CTL (CNRM) Z10

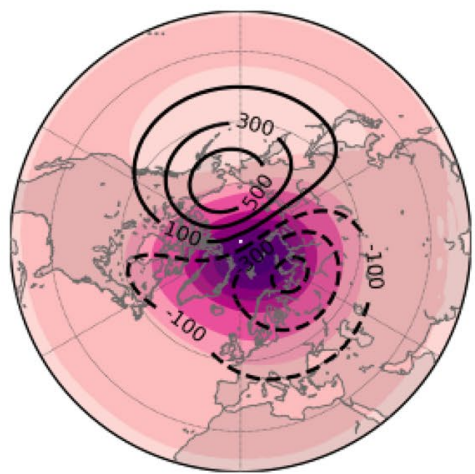

(h) CTL (CNRM) Z50

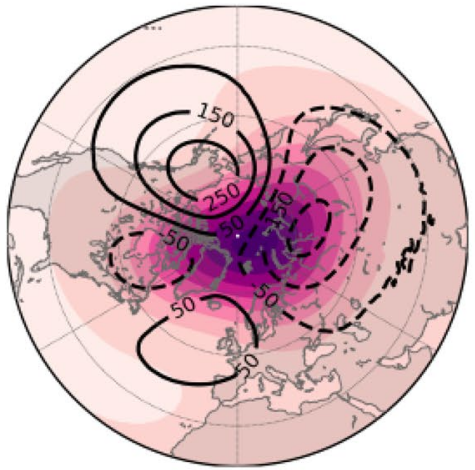

(k) CTL (CNRM) Z100

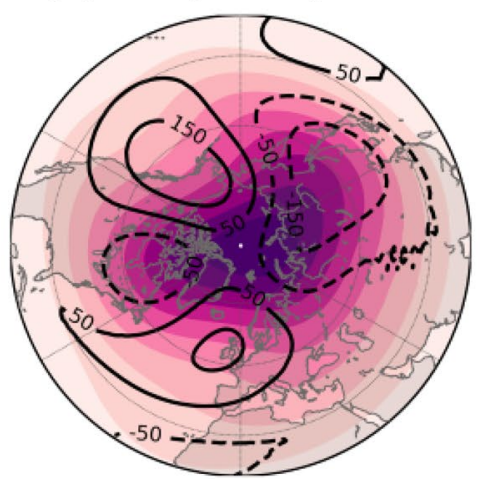

(c) CTL (CMCC) Z1

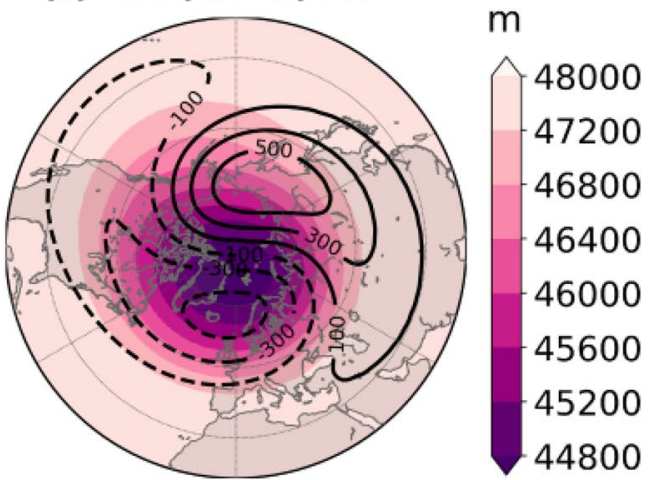

(f) CTL (CMCC) Z10

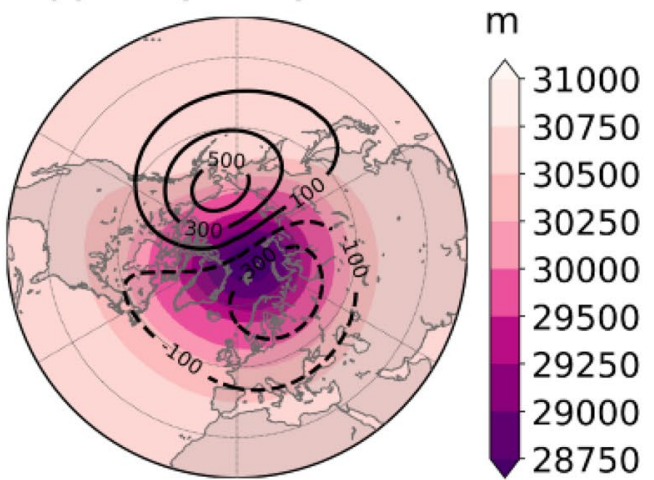

(i) CTL (CMCC) Z50

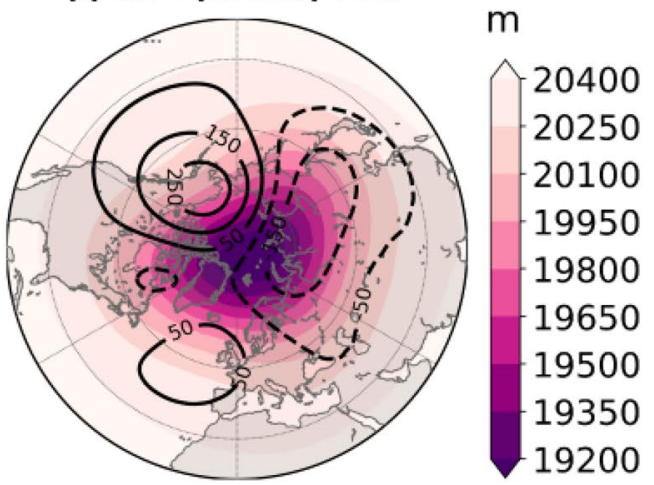

(I) CTL (CMCC) Z100

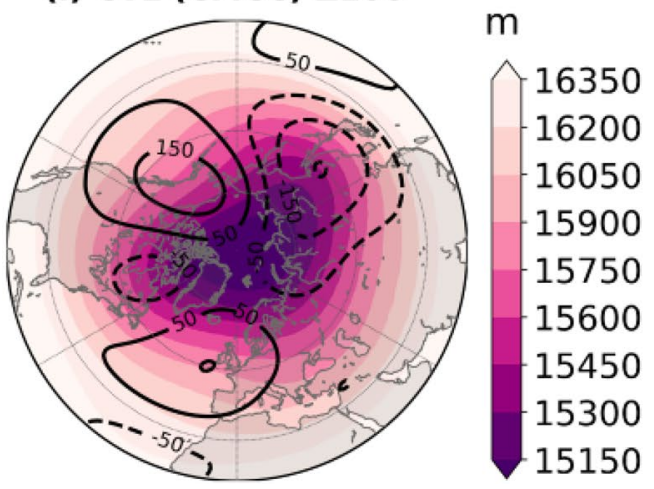

Fig. 2 JFM climatology of geopotential height (shading) and zonaleddy geopotential height (contours) from CTL at $1 \mathrm{hPa}$ (first row), $10 \mathrm{hPa}$ (second row), $50 \mathrm{hPa}$ (third row) and $100 \mathrm{hPa}$ (fourth row):
EC-EARTH (left), CNRM (middle), CMCC (right). Contour interval: $\pm 100, \pm 300, \pm 500$ in the first two rows; $\pm 50, \pm 150, \pm 250$ in the two bottom rows 
(a) EN (EC-EARTH) JFM [U]

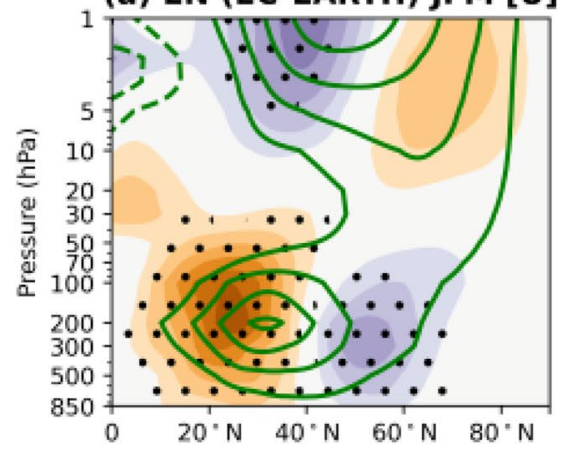

(d) EN (EC-EARTH) JFM [T]

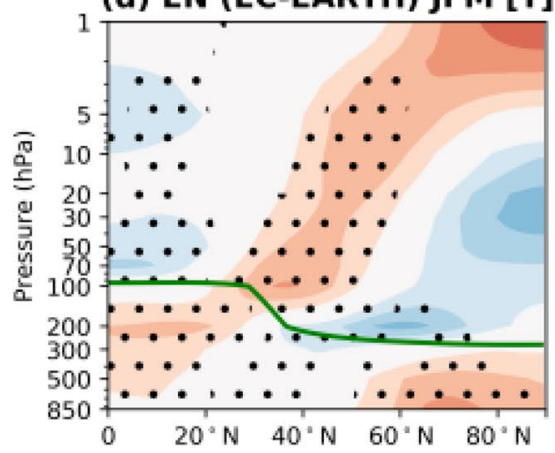

(g) LN (EC-EARTH) JFM [U]
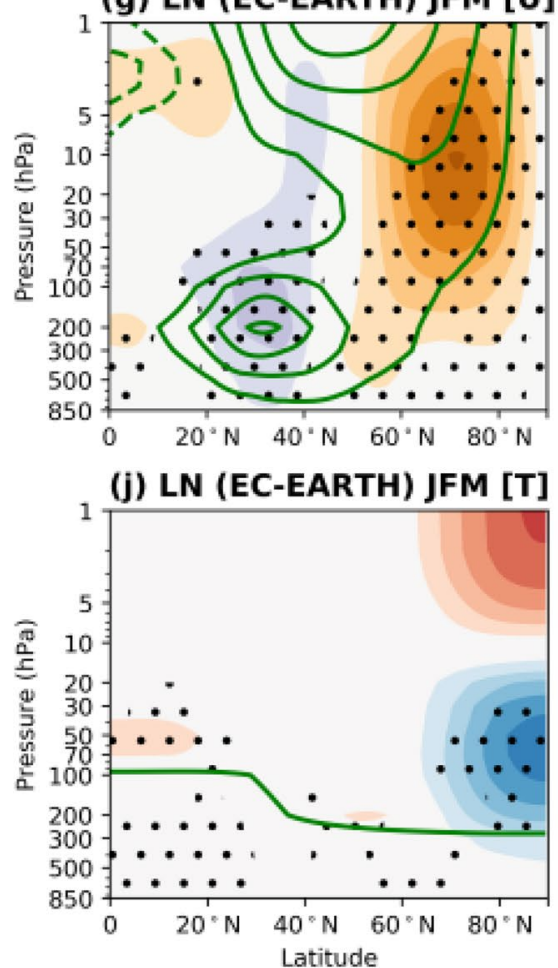

(b) EN (CNRM) JFM [U]

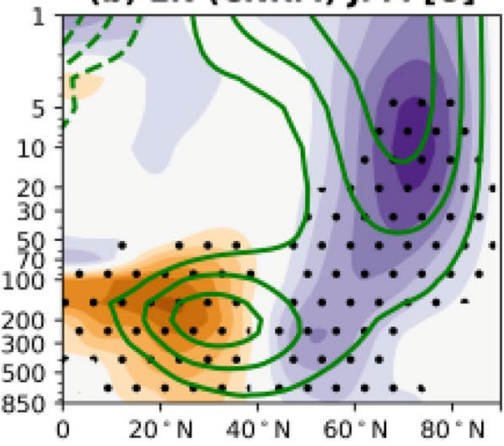

(e) EN (CNRM) JFM [T]

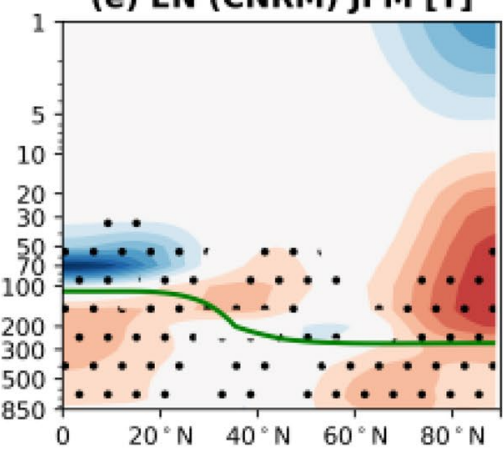

(h) LN (CNRM) JFM [U]

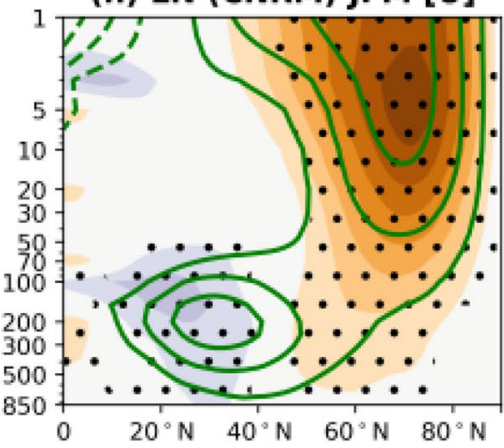

(k) LN (CNRM) JFM [T]

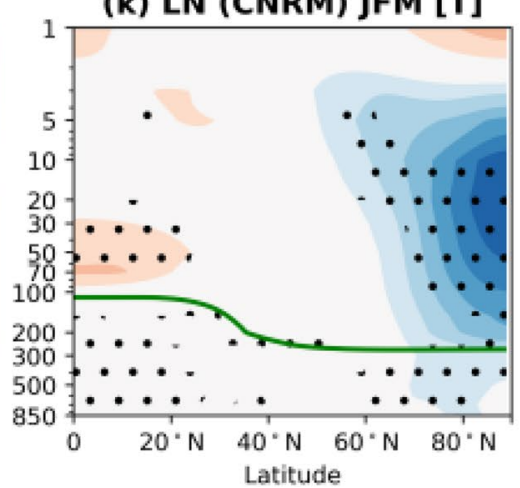

(c) EN (CMCC) JFM [U] $\mathrm{ms}^{-1}$

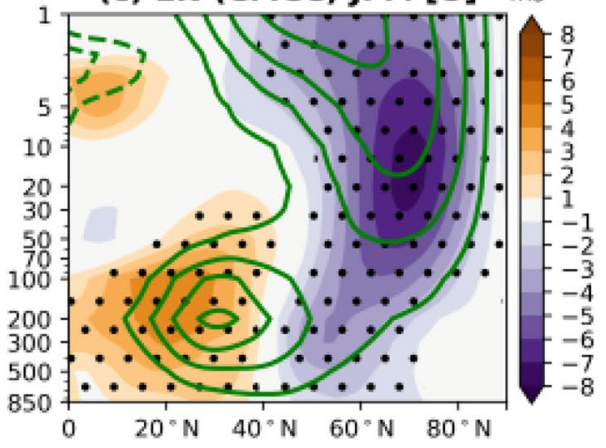

(f) EN (CMCC) JFM [T] ${ }^{\circ} \mathrm{C}$

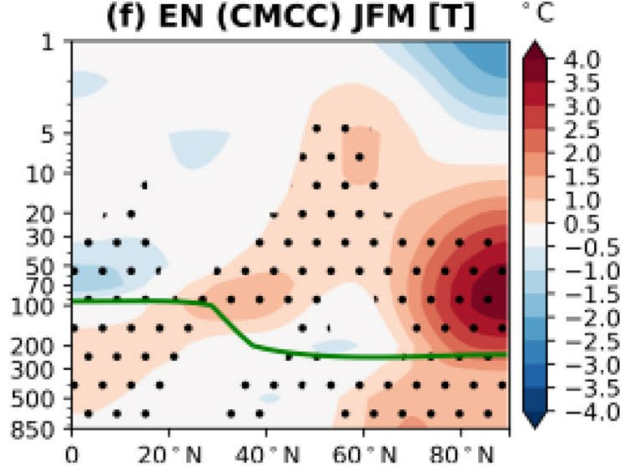

(i) LN (CMCC) JFM [U] $\mathrm{ms}^{-1}$

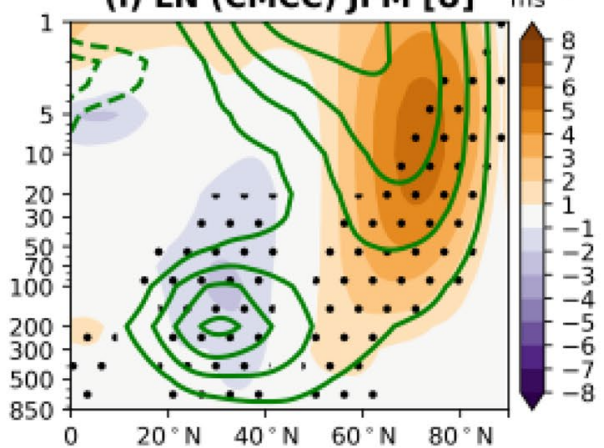

(I) LN (CMCC) JFM [T]

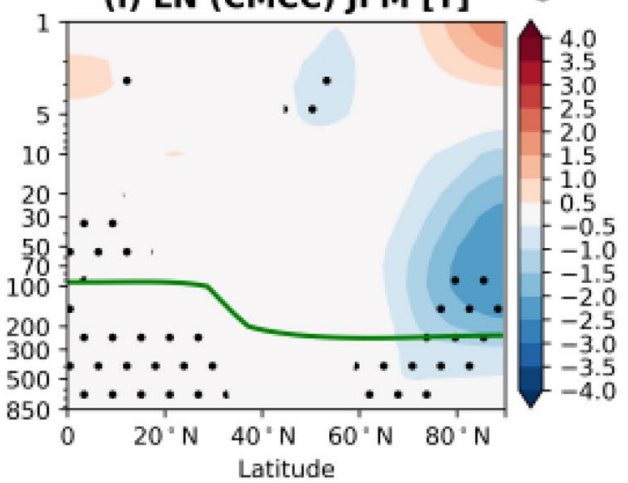

Fig. 3 a-f Ensemble-mean zonal-mean zonal wind (top row) and temperature (second row) anomalies for EN with respect to CTL in JFM: EC-EARTH (left), CNRM (middle), CMCC (right). g-l Same as (a-f), but for LN. Green contours in the zonal wind panels show its climatology from CTL (contour interval: $10 \mathrm{~ms}^{-1}$ ) and the green line in the temperature panels depicts the tropopause level from CTL, according to the WMO's definition (lowest level at which the lapse rate decreases to $2{ }^{\circ} \mathrm{C} / \mathrm{km}$ or less). Dotted areas indicate statistical significance at the $95 \%$ confidence level 
(a) EN (EC-EARTH) JF

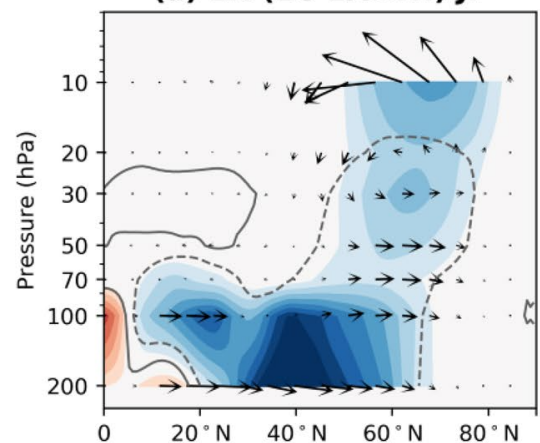

(d) LN (EC-EARTH) JF

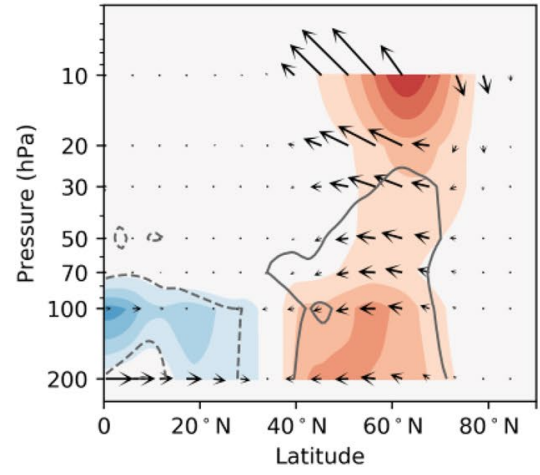

(b) EN (CNRM) JF

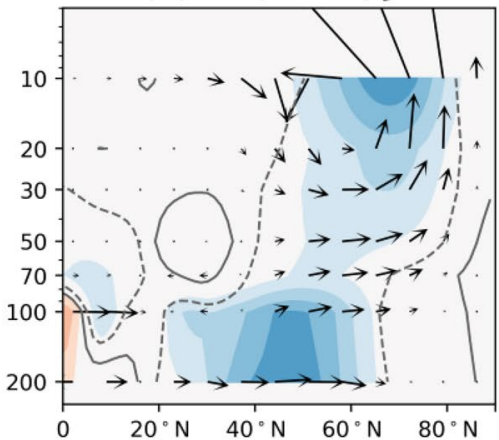

(e) LN (CNRM) JF

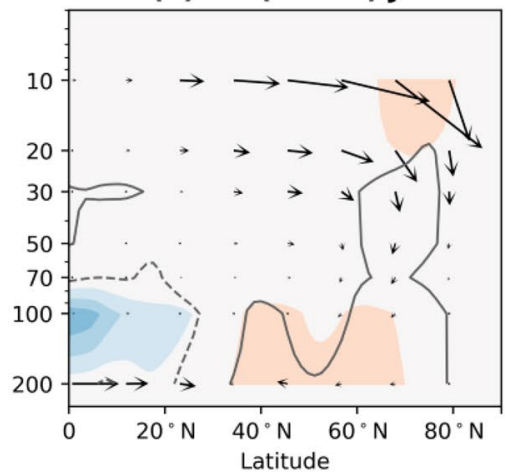

(c) EN (CMCC) JF
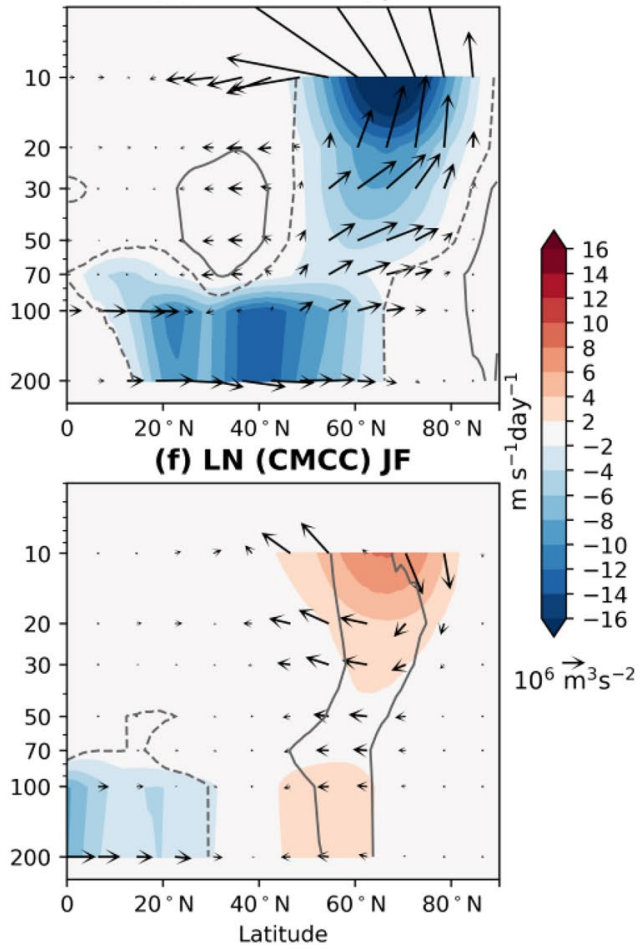

Fig. 4 Top: EP flux (arrows) and EP-flux divergence (shading) anomalies for EN with respect to CTL in JF: EC-EARTH (left), CNRM (middle), CMCC (right). Bottom: same as Top, but for LN. The EP flux is shown as $F / \rho a$ and scaled by $\sqrt{1000 / p}$ as in Taguchi and
Hartmann (2006); to improve visibility, $F_{z}$ is multiplied by 100 , as in Rao and Ren (2016a). Black contours (solid for positive, dashed for negative anomalies) indicate statistical significance at the $95 \%$ confidence level for the EP-flux divergence
EN. To explore this possibility, we examine the anomalous EP flux and its divergence (Fig. 4; see Sect. 2.2). Although the wave driving of the stratospheric circulation might be almost instantaneous (e.g. Shaw et al. 2014), we only consider JF, as in previous studies, as that is when the ENSOrelated anomalous wave injection is expected to peak (e.g. García-Herrera et al. 2006; Bell et al. 2009). We begin by analyzing the divergence of the EP flux, $\nabla \cdot F$ (Fig. 4, shading), recalling that negative (positive) anomalies indicate divergence (convergence) of zonal momentum $\left(u^{*} v^{*}\right)$ and deceleration (acceleration) of the flow. For EN (Fig. 4, top), a dominant negative $\nabla \cdot F$ anomaly is present in all cases throughout the atmosphere, in agreement with previous studies (e.g. Taguchi and Hartmann 2006; Trascasa-Castro et al. 2019), but with differences in magnitude and significance. In particular, all models show maximum EP-flux convergence in mid-latitudes at lower levels (below $70 \mathrm{hPa}$ ), consistent with the weakening of the tropospheric jet at its poleward flank (Fig. 3, top), observed even in EC-EARTH (Fig. 3a). Another negative maximum is present at higher latitudes in the upper levels $(\sim 10 \mathrm{hPa})$, which is consistent with the middle stratospheric anomalies in zonal-mean zonal wind in CMCC and CNRM (Fig. 3b, c) but inconsistent with the (lack of) signal in EC-EARTH (Fig. 3a). This inconsistency suggests that anomalous wave breaking may not actually drive the weakening of the polar vortex in these models. This hypothesis is further supported by the anomalous EP flux divergence in LN (Fig. 4g-i), which is qualitatively symmetric to that in EN, in agreement with previous studies (e.g. Iza et al. 2016; Trascasa-Castro et al. 2019), but much weaker and less significant, particularly in CNRM (Fig. 4e) even though that is the model with the largest strengthening of the polar vortex (Fig. 3h).

The vertical component of the EP flux $\left(F_{z}\right)$, which is proportional to $\left[v^{*} T^{*}\right]$, is related to the vertical propagation of wave activity, and thus upward (downward) pointing vectors suggest enhanced (reduced) wave propagation towards higher levels. In EN, all models show EP-flux vectors pointing poleward in the lower stratosphere (up to $50 \mathrm{hPa}$; recall that $F_{y}$ is proportional to $\left.-\left[u^{*} v^{*}\right]\right)$, and again behave similarly at $10 \mathrm{hPa}$, where they tend to turn equatorward (Fig. 3a-c). Between 50 and $10 \mathrm{hPa}$, the EP-flux vectors in CNRM and CMCC turn upwards at high latitudes (north of $60^{\circ}-70^{\circ} \mathrm{N}$ ), while the vertical component of the EP flux seems negligible in EC-EARTH, a difference that we will further discuss in Sect. 3.6. In LN, the EPflux vectors do not clearly indicate a suppression of wave 
propagation, except in the upper levels and at high latitudes (Fig. 4, bottom).

\subsection{Longitude-latitude structure of temperature anomalies in the lower stratosphere}

While zonal-mean profiles are a common approach to examine the vertical structure of the ENSO anomalies, looking at the spatial distribution of the signal in the longitude-latitude plane at some key levels is convenient to gain further insight into the models' response (e.g. Baldwin and O'Sullivan
1995; Sassi et al. 2004; Manzini et al. 2006; Garfinkel and Hartmann 2007; Cagnazzo and Manzini 2009). Due to the wave filtering exerted by strong westerly winds, different anomalous patterns are expected in the lower and middleupper stratosphere, similarly to what is seen in the climatology (see Sect. 3.2): for this reason, here we show maps of anomalous temperature at 200, 100 and $50 \mathrm{hPa}$ (Figs. 5 and 6), while the response above $50 \mathrm{hPa}$ is discussed in Sect. 3.6. Note that in mid-latitudes and further north, the $200 \mathrm{hPa}$ level is located above the tropopause (see green contour in Fig. 3). For EN, the lowermost stratosphere shows (a) EN (EC-EARTH) T50

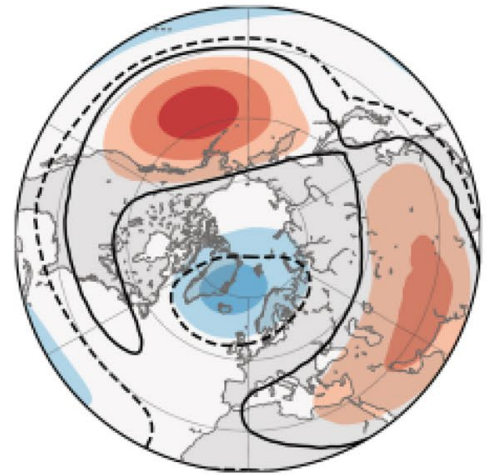

(d) EN (EC-EARTH) T100

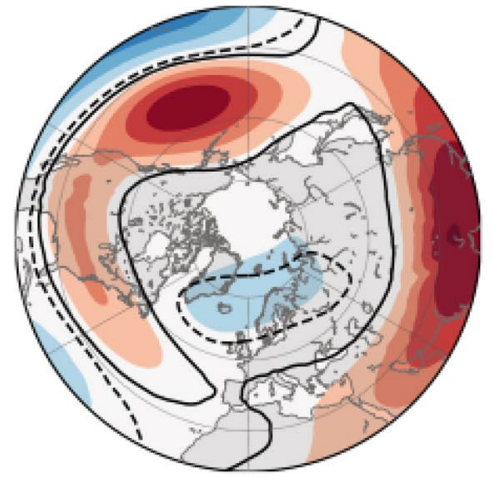

(g) EN (EC-EARTH) T200

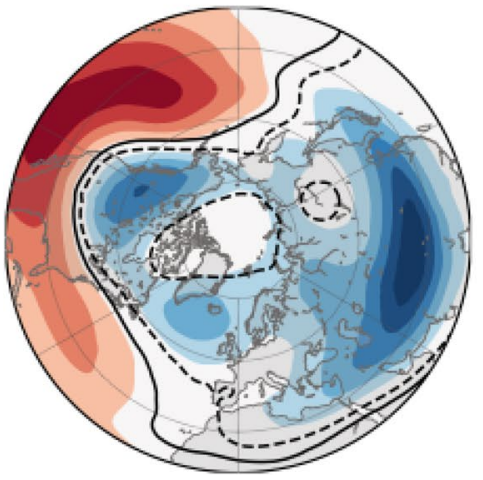

(b) EN (CNRM) T50

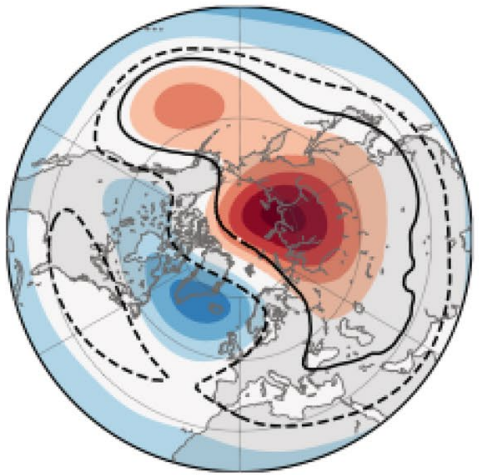

(e) EN (CNRM) T100

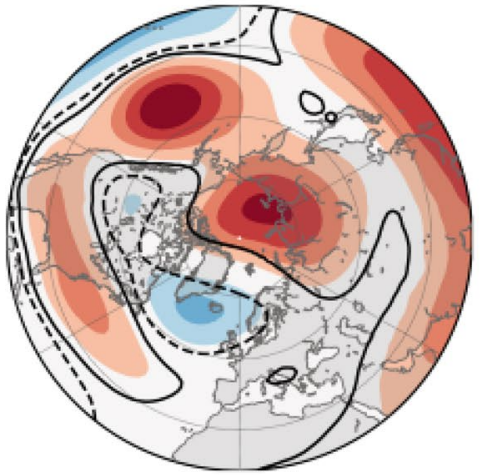

(h) EN (CNRM) T200

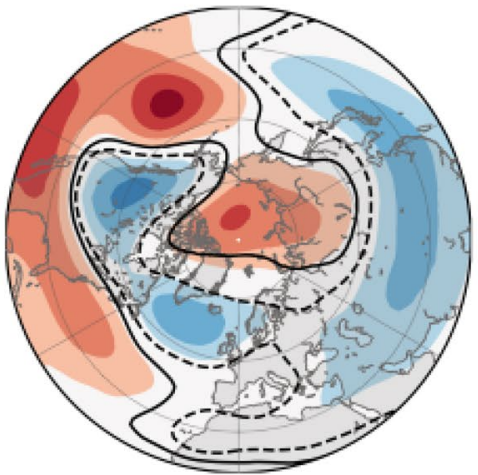

(c) EN (CMCC) T50

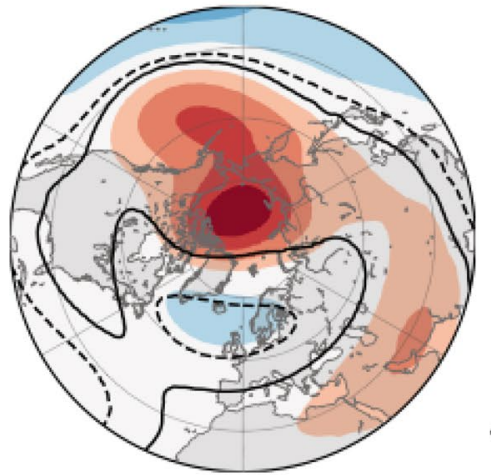

(f) EN (CMCC) T100
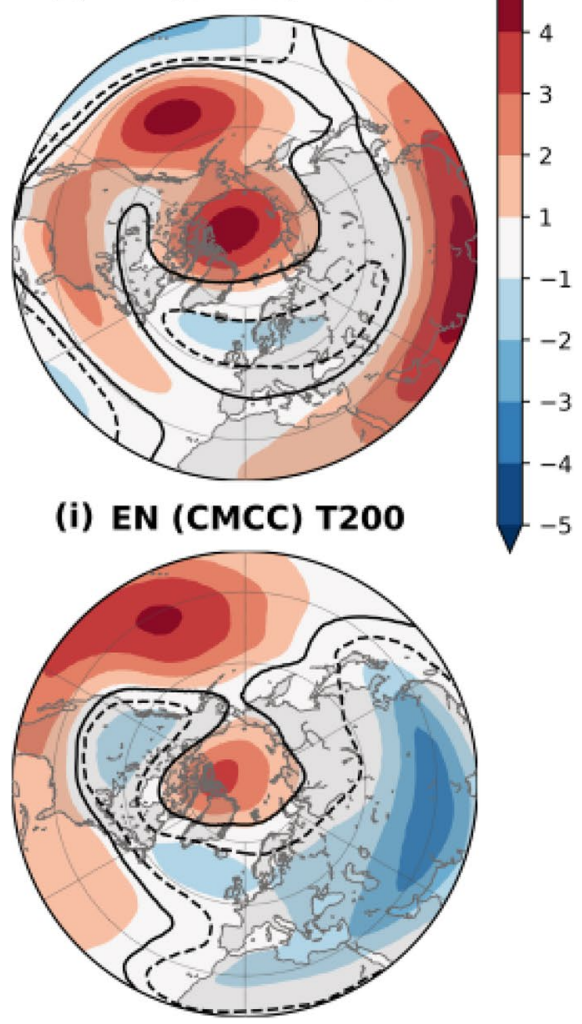

Fig. 5 Ensemble-mean temperature anomalies for EN with respect to CTL in JFM at 50 (top), 100 (middle) and $200 \mathrm{hPa}$ (bottom): ECEARTH (left), CNRM (middle), CMCC (right). Black contours (solid for positive, dashed for negative anomalies) indicate statistically significant areas at the $95 \%$ confidence level 
(a) LN (EC-EARTH) T50

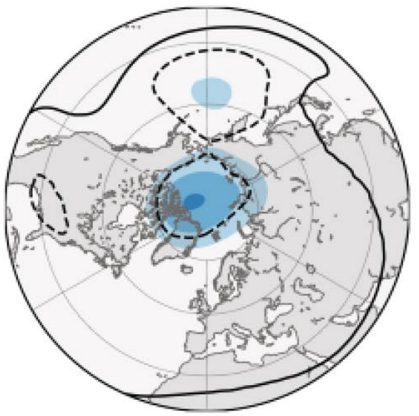

(d) LN (EC-EARTH) T100

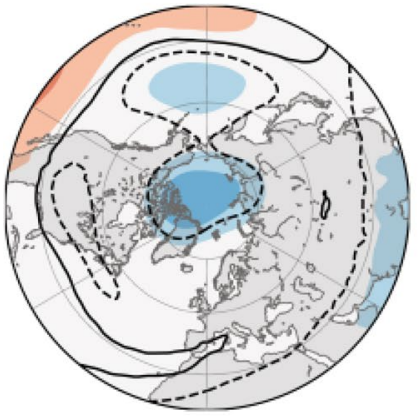

(g) LN (EC-EARTH) T200

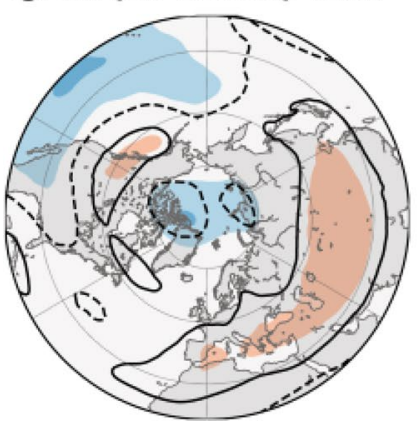

(b) LN (CNRM) T50

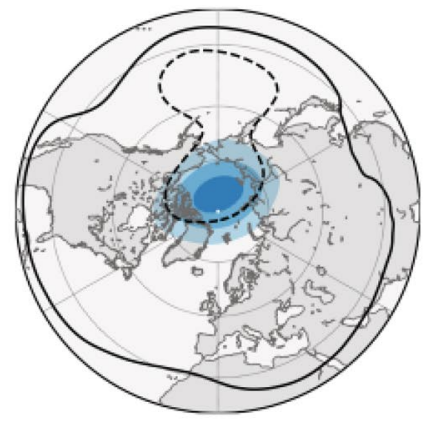

(e) LN (CNRM) T100

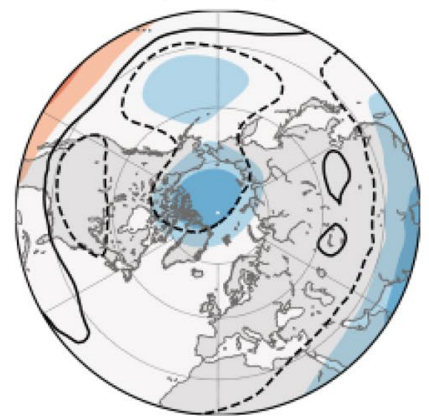

(h) LN (CNRM) T200

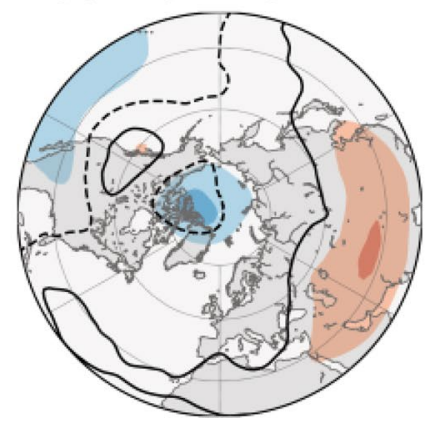

(c) LN (CMCC) T50
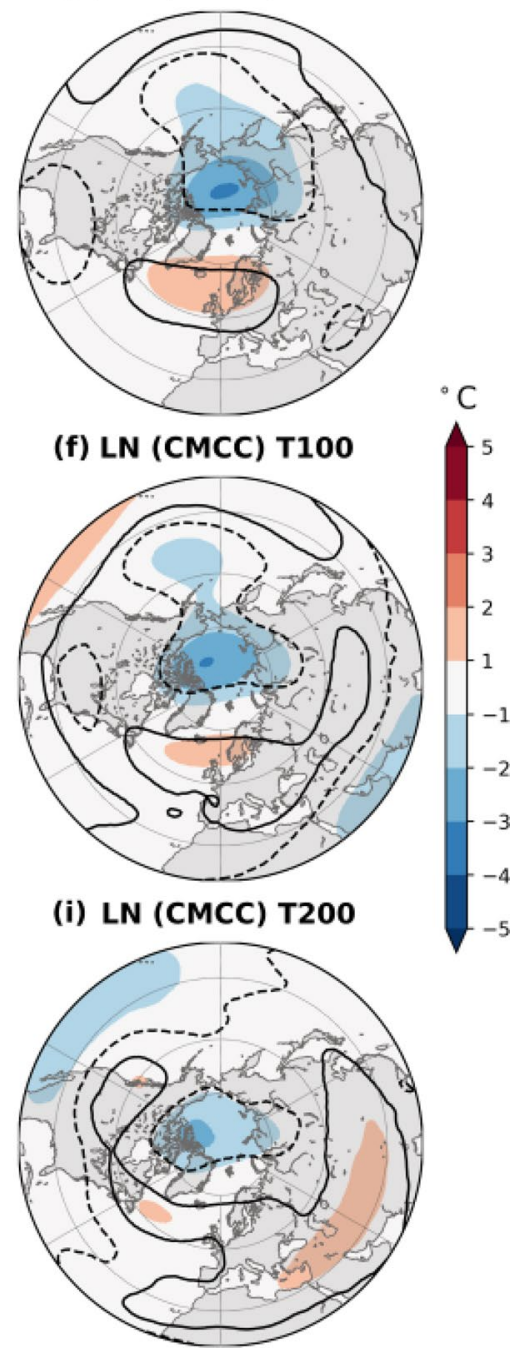

Fig. 6 Ensemble-mean temperature anomalies for $\mathrm{LN}$ with respect to CTL in JFM at 50 (top), 100 (middle) and $200 \mathrm{hPa}$ (bottom): ECEARTH (left), CNRM (middle), CMCC (right). Black contours (solid

a cooling at subpolar latitudes over North America and the North Atlantic (Fig. 5g-i). The peak in the warming of the polar cap in a zonal-mean sense is found around $100 \mathrm{hPa}$ (Fig. 3b, c), but a positive temperature anomaly over polar latitudes is already present at $200 \mathrm{hPa}$ in CMCC and CNRM, albeit displaced towards the North Pacific sector (Fig. 5h, i). While the subpolar cooling is also present in EC-EARTH, the warming over the polar cap is strikingly absent (Fig. 5g), indicating that this model is not simulating a displaced or weaker response masked by the zonal mean but is not yielding a response over the polar cap at all. The same situation is seen at higher levels, where CNRM and CMCC show an even stronger polar warming (Fig. 5b, c, e, and f), while ECEARTH lacks any significant signal in that region (Fig. 5a, d). Note that, in contrast, no discrepancy is found between the models in the troposphere (Fig. S1). For LN (Fig. 6), for positive, dashed for negative anomalies) indicate statistically significant areas at the $95 \%$ confidence level

instead, and as with the zonal mean (Fig. 3, bottom row), the temperature response is similar across the models, with the main feature being the cooling over the polar cap between 200 and $50 \mathrm{hPa}$, which, as in the case of EN, is located in the North Pacific sector (Fig. 5d-i). The temperature anomalies are weaker than in EN (by 25\% for the polar anomaly) but significant, even in EC-EARTH. In the observational composites, the polar cap anomalies appear more zonally symmetric (Fig. 11d), but the statistical significance for La Niña is limited to the North Pacific sector (Fig. 11h).

\subsection{Longitude-latitude structure of geopotential height anomalies in the lower stratosphere}

To gain further insight into the lower stratospheric response, we now examine maps of anomalous geopotential height 
(a)EN(EC-EARTH) Z50

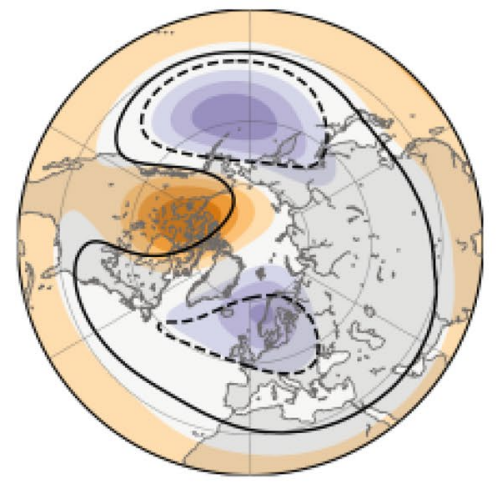

(d)EN(EC-EARTH) Z100

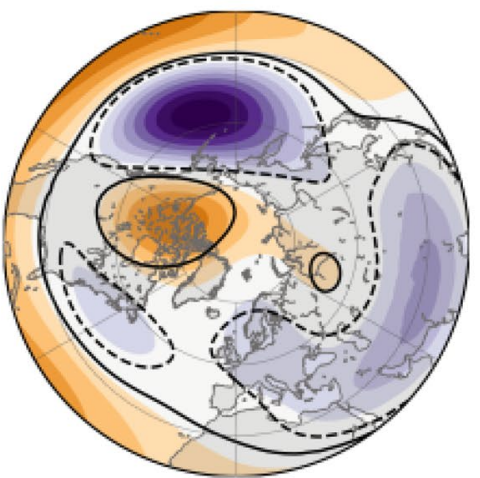

(g)EN(EC-EARTH) Z200

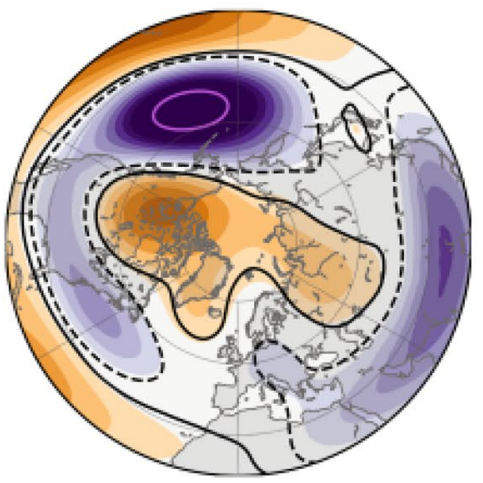

(b)EN(CNRM) Z50

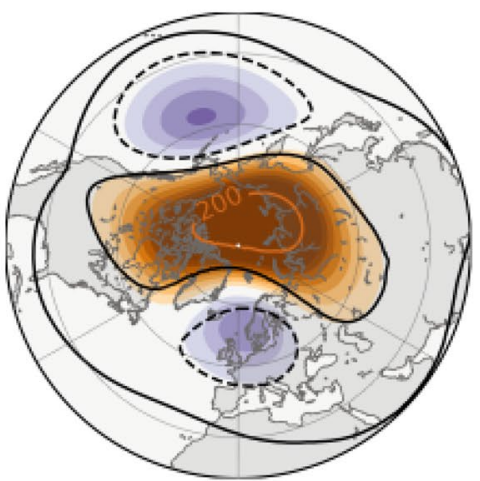

(e)EN(CNRM) Z100

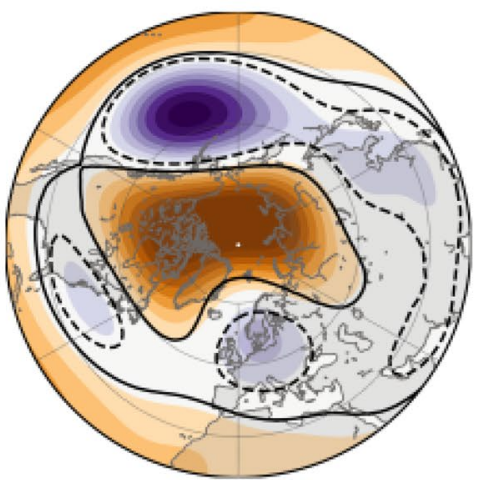

(h)EN(CNRM) Z200

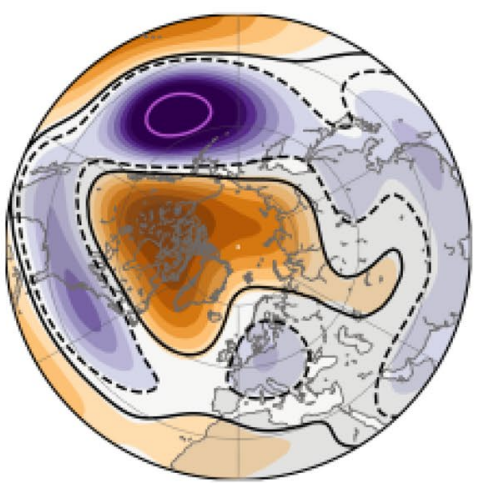

(c)EN(CMCC) Z50

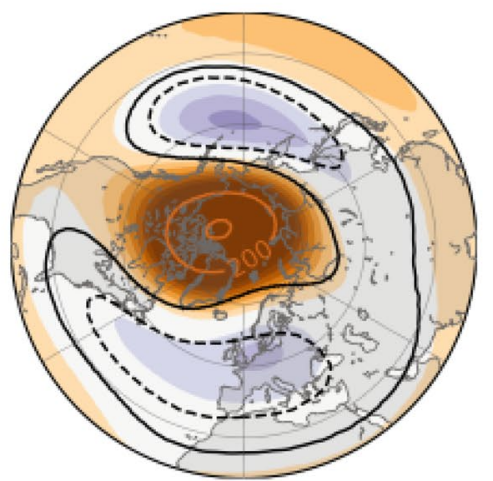

(f)EN(CMCC) Z100
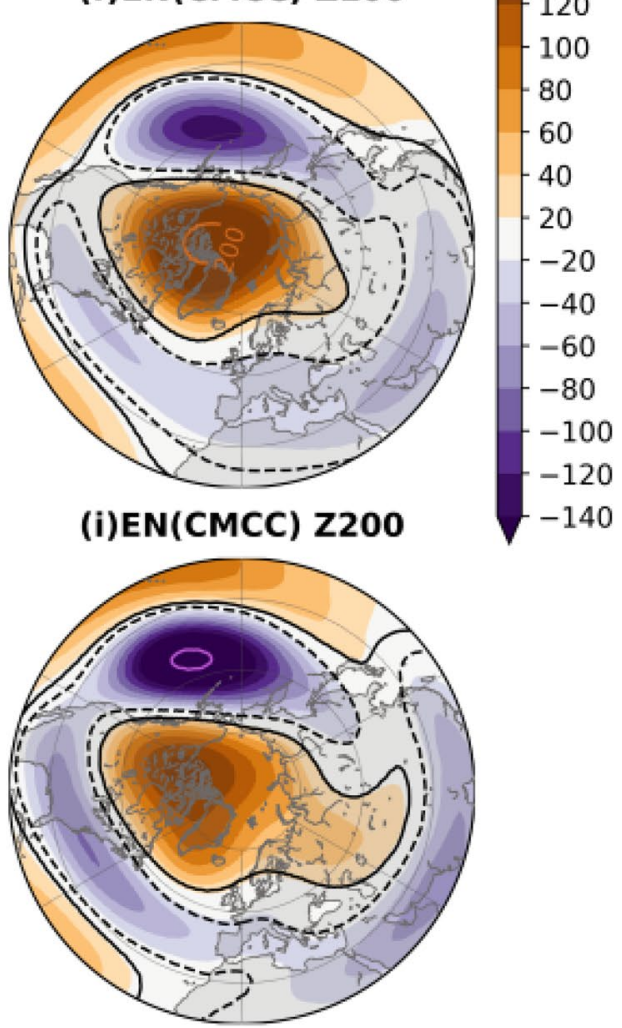

Fig. 7 Ensemble-mean geopotential height anomalies for $\mathrm{EN}$ with respect to CTL in JFM at 50 (top), 100 (middle) and $200 \mathrm{hPa}$ (bottom): EC-EARTH (left), CNRM (middle), CMCC (right). Orange and magenta contours show values exceeding the color scale limit at \pm 200 , and $\pm 300 \mathrm{~m}$. Black contours (solid for positive, dashed for negative anomalies) indicate statistically significant areas at the $95 \%$ confidence level

anomalies, placed in the North Pacific sector (Fig. 5b, c, e, $\mathrm{f}, \mathrm{h}$, and i). The anomalous anticyclonic circulation associated with this high-pressure system over the polar cap is also dynamically consistent with the weakening of the lower stratospheric westerly winds (Fig. 3b, c), in accordance with quasi-geostrophic balance. In EC-EARTH, by contrast, this positive center of action has smaller amplitude already at $200 \mathrm{hPa}$ (Fig. 7g). More importantly, while it is centered over Canada as in the other two models, it only weakly extends towards the pole. At upper levels, it further weakens north of $70^{\circ} \mathrm{N}$ (Fig. 7b, c) and again, like the temperature 
(a) LN(EC-EARTH) Z50

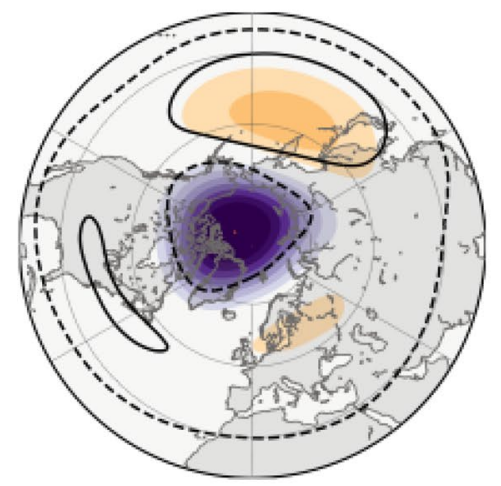

(d) LN (EC-EARTH) Z100

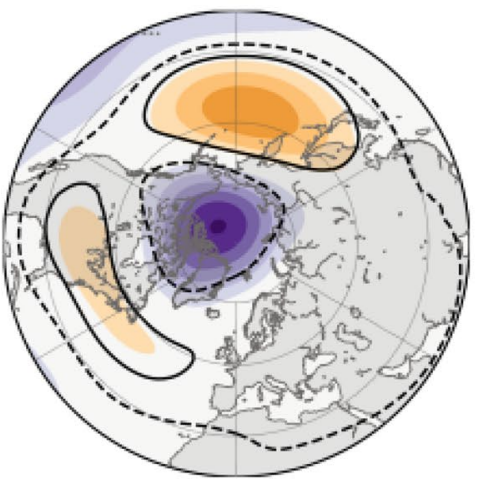

(g)LN(EC-EARTH) Z200

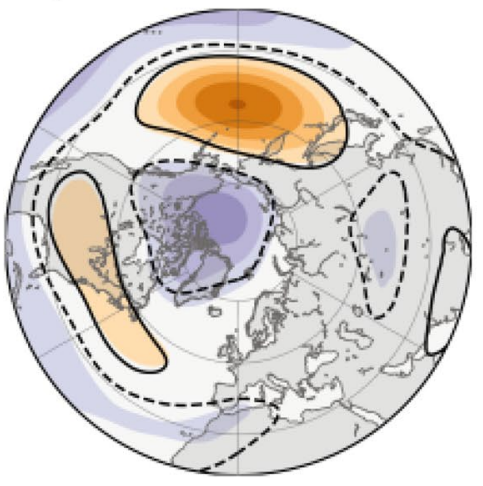

(b)LN(CNRM) Z50

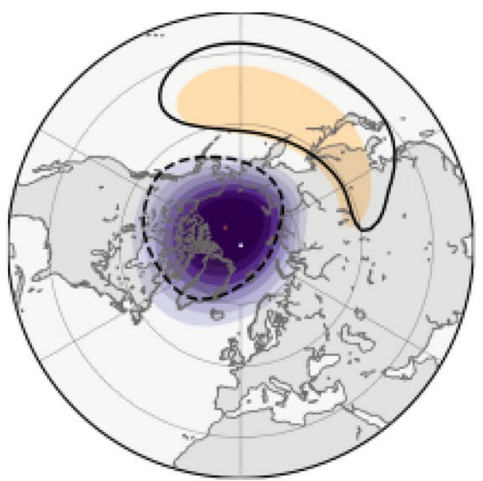

(e)LN(CNRM) Z100

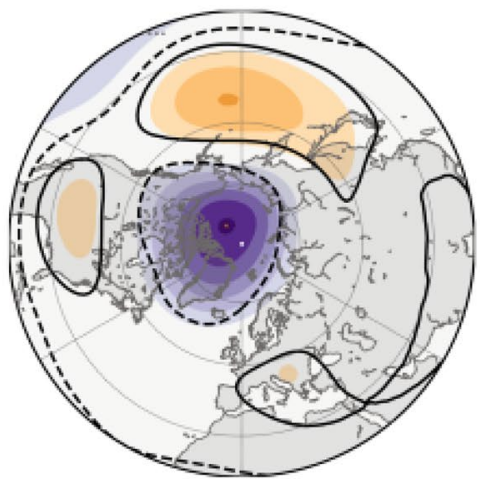

(h)LN(CNRM) Z200

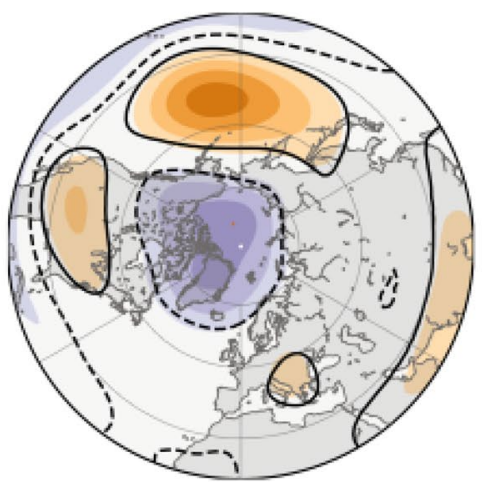

(c)LN (CMCC) Z50

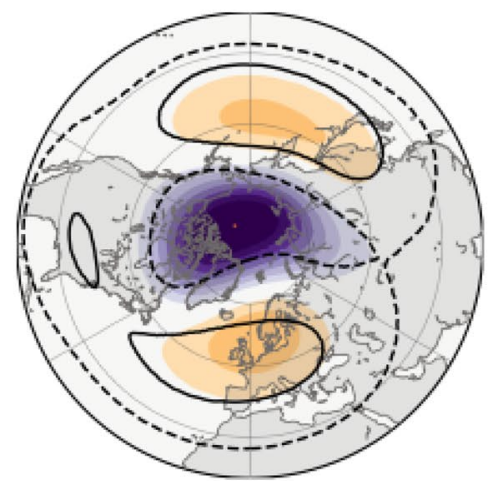

(f)LN(CMCC) Z100

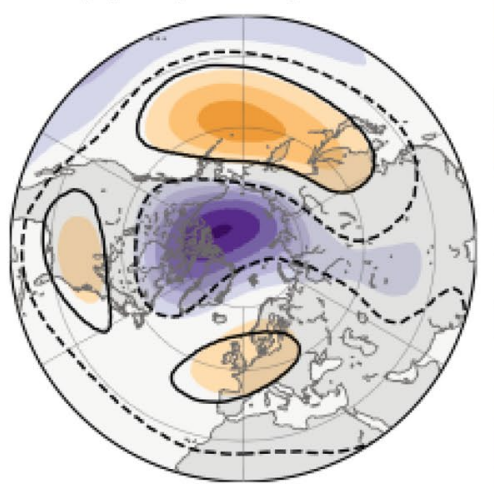

(i)LN(CMCC) Z200

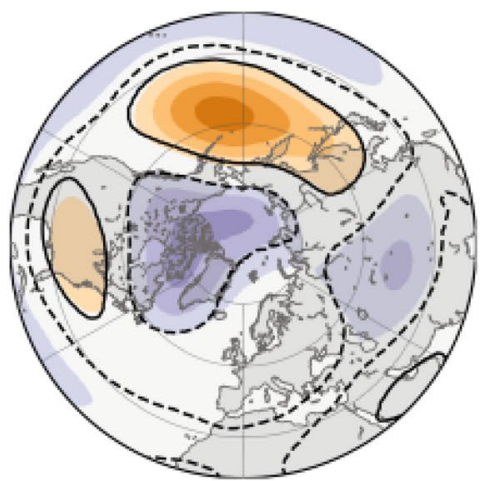

Fig. 8 Ensemble-mean geopotential height anomalies for LN with respect to CTL in JFM at 50 (top), 100 (middle) and $200 \mathrm{hPa}$ (bottom): EC-EARTH (left), CNRM (middle), CMCC (right). Black con- tours (solid for positive, dashed for negative anomalies) indicate statistically significant areas at the $95 \%$ confidence level

stratosphere it is mostly superimposed to positive values of the climatological stationary wave pattern, preventing linear constructive interference over the mid-latitude eastern North Pacific (cf. Figures 7 and 2g-1). In the North Atlantic sector, the dipole-like pattern near the surface (Fig. 1a-c) extends to only $200 \mathrm{hPa}$ (Fig. $7 \mathrm{~g}-\mathrm{i}$ ), with only the southern lobe remaining at $100 \mathrm{hPa}$ and disappearing beyond that.

Similarly to what was observed for temperature, ECEARTH largely agrees with the other models for LN (Fig. 8). The forced wave train tends to be shifted westward in mid-latitudes compared to its EN counterpart (Mezzina 
et al. 2021) and the negative anomaly over Canada peaks at higher latitudes, i.e., north of $70^{\circ} \mathrm{N}$ (cf. bottom rows in Figs. 7 and 8). It amplifies with increasing altitude but maintains its structure, largely covering the polar cap (Fig. 8a-f), although slightly displaced towards the North Pacific sector, like the temperature anomalies (Fig. 6a-f). The anomalous cyclonic circulation associated with this low-pressure system over the polar cap is again consistent with the strengthening of the zonal-mean westerly winds at lower stratospheric levels (Fig. 3h-i), according to quasi-geostrophic balance. As for temperature, weak significance is found in the observational composite for La Niña (Fig. 11f), in line with the zonal-mean zonal wind response (Fig. 11e).

We have found that EC-EARTH is able to capture the expected geopotential height response for $\mathrm{LN}$, but not for $\mathrm{EN}$, in contrast to the other models (which behave symmetrically). While this model is not completely failing in simulating the anomalous high over Canada in EN, the lack of positive geopotential height anomalies over the polar cap in the lower stratosphere is consistent with the wrong zonal wind response and appears to be related to the missing warming of the lower stratosphere.

\subsection{ENSO signal in the middle-upper stratosphere}

We now turn to the middle-upper stratosphere and examine in detail the circulation $(Z)$ response to ENSO above $50 \mathrm{hPa}$. For EN (Fig. 9), CNRM and CMCC show already at $10 \mathrm{hPa}$ a pattern with a strong WN1 component, which dominates at all levels (Fig. 9, middle and right columns). This response appears to be related to the lower-stratospheric anomalies, which propagate upwards, as suggested by the westward tilt with height, and adopt this predominantly WN1 structure due to the stronger westerly winds and the Charney-Drazin wave filtering (Fig. 2; see Sect. 3.1). In these two models, the geopotential height anomalies, which grow in amplitude with increasing height (cf. Figures 9 and 7), are largely in phase with the climatological wave pattern at all levels. In particular, the positive anomaly strengthens the stratospheric Aleutian High, while the negative one reinforces the cyclonic circulation associated with the vortex displacement in the North Atlantic sector (cf. Figs. 9 and 2): the net result is a weakening of the vortex (Fig. 3b, c) and a displacement towards Greenland (e.g. Harvey and Hitchman 1996; Harvey et al. 2002; Nigam and DeWeaver 2003). In EC-EARTH, in contrast, the $10 \mathrm{hPa}$ pattern is more reminiscent of a WN2 structure, related to the distinct lower stratospheric response and to the weaker climatological winds between 50 and $10 \mathrm{hPa}$ (Fig. 3a), which do not effectively filter out smaller components. At 5 and $1 \mathrm{hPa}$, the winds are strong enough for the WN1 component to become dominant (Figs. 3a, 9a, d) and the response is more similar to that of the other two models, again showing a strengthening of the stratospheric Aleutian High. Note, however, that the anomalies barely reach the polar cap, which is consistent with the erroneous response in the zonal-mean zonal wind at high latitudes (Fig. 3a).

The westward tilt with height of the anomalies in the middle-upper stratosphere is a common aspect to all models (Fig. 9) that indicates upward propagation of (stationary) wave activity, which is in turn associated with positive anomalous $v^{*} T^{*}$ (see Sect. 2.2). This would appear to be in contrast with the lack of a vertical EP-flux component in ECEARTH between 50 and $10 \mathrm{hPa}$ (Fig. 4a); we note, however, that anomalous positive $v^{*} T^{*}$ is present at $50 \mathrm{hPa}$ outside the polar cap (Fig. 12d), cancelling out in the zonal mean. It is only at $10 \mathrm{hPa}$ that anomalous positive $v^{*} T^{*}$ appears at high latitudes, north of $70^{\circ} \mathrm{N}$, and dominates the zonal mean (Fig. 12a), consistent with the EP-flux vectors and in agreement with CNRM and CMCC (Figs. 4, 12). Therefore, there is upward wave propagation from the lowermost throughout the middle-upper stratosphere in all models, including EC-EARTH.

As before, for LN there is more consistency across the models (Fig. 10), although CMCC shows a stronger WN1 pattern that is symmetric in structure to that in EN (cf. Figs. 9 and 10, right column): here, the anomalies are out of phase with the climatological stationary eddy field and the stratospheric Aleutian High is weakened, thereby the vortex is reinforced and shifted towards the North Pacific sector (cf. Figs. 10 and 2, right columns). While it is less evident, in EC-EARTH and CNRM, the cyclonic anomaly is also offcentered with respect to the pole, towards the North Pacific (Fig. 10, left and middle columns), hence also destructively interfering with the climatological Aleutian High (see Fig. 2). This overall WN1 component of the response to La Niña in the upper stratosphere (see also Mezzina et al. 2021) is in agreement with the model results of Manzini et al. (2006). In all models, the westward tilt with height is again evident, but less pronounced than for EN. While the amplitude of the circulation anomalies slightly increases with height from the lower (Fig. 8) to the middle stratosphere, they weaken and lose statistical significance at the stratopause (1 hPa), particularly in EC-EARTH (Fig. 10a) and CMCC (Fig. 10c), which may be indicative of wave dissipation at this level.

Note that the response in $\mathrm{LN}$ is again weaker than in EN, highlighting the importance of examining longitude-latitude maps of the anomalies at some key levels to complement the zonal-mean analysis, which instead showed a similar amplitude for EN and LN in CNRM (see Sect. 3.2), due to the WN1 character of the response. This is also true for the temperature anomalies, which appear to be confined to the lower stratosphere in the zonal mean (Fig. 3), but are in 
(a)EN (EC-EARTH) Z1

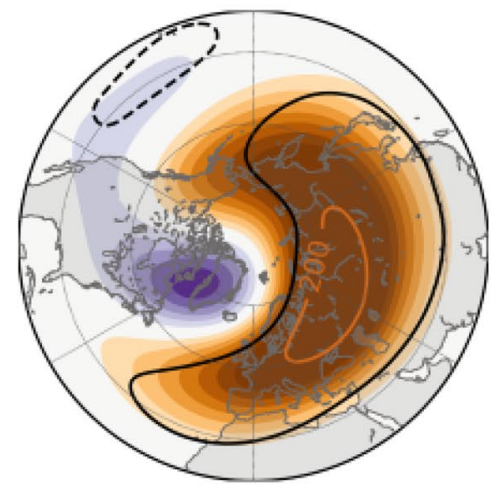

(d)EN (EC-EARTH) Z5

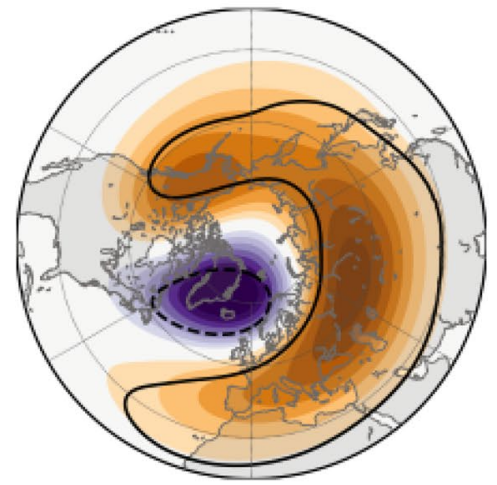

(g)EN (EC-EARTH) Z10

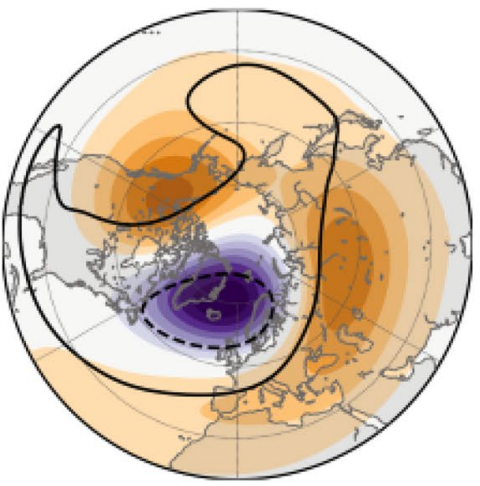

(b)EN (CNRM) Z1

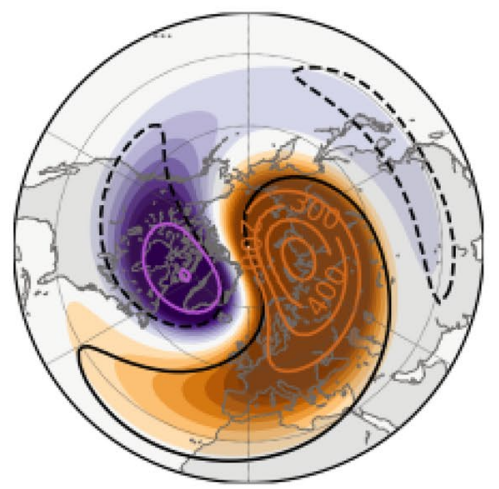

(e)EN (CNRM) Z5

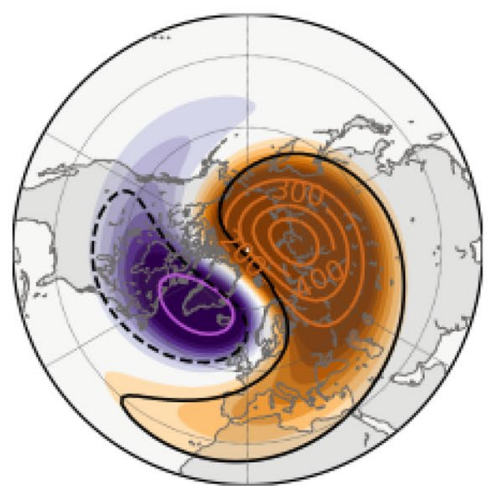

(h)EN (CNRM) Z10

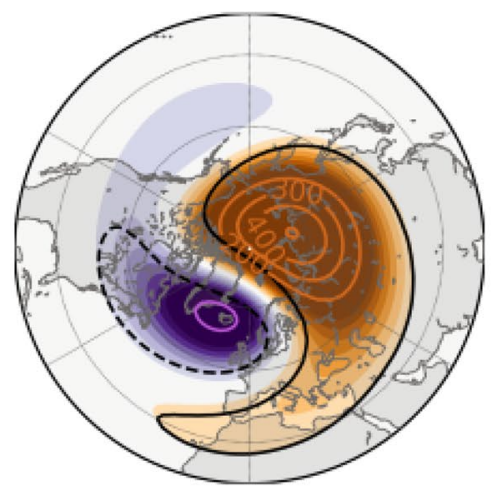

(c)EN (CMCC) Z1

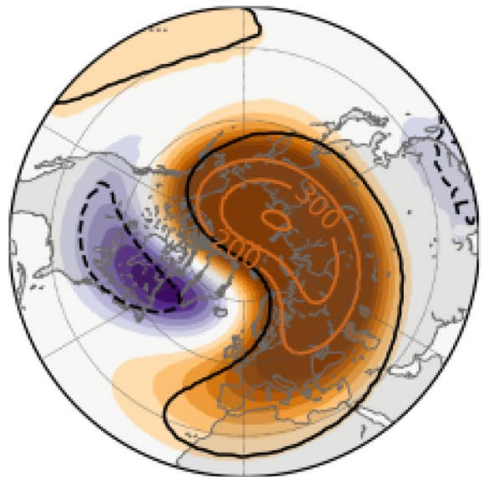

(f)EN (CMCC) Z5
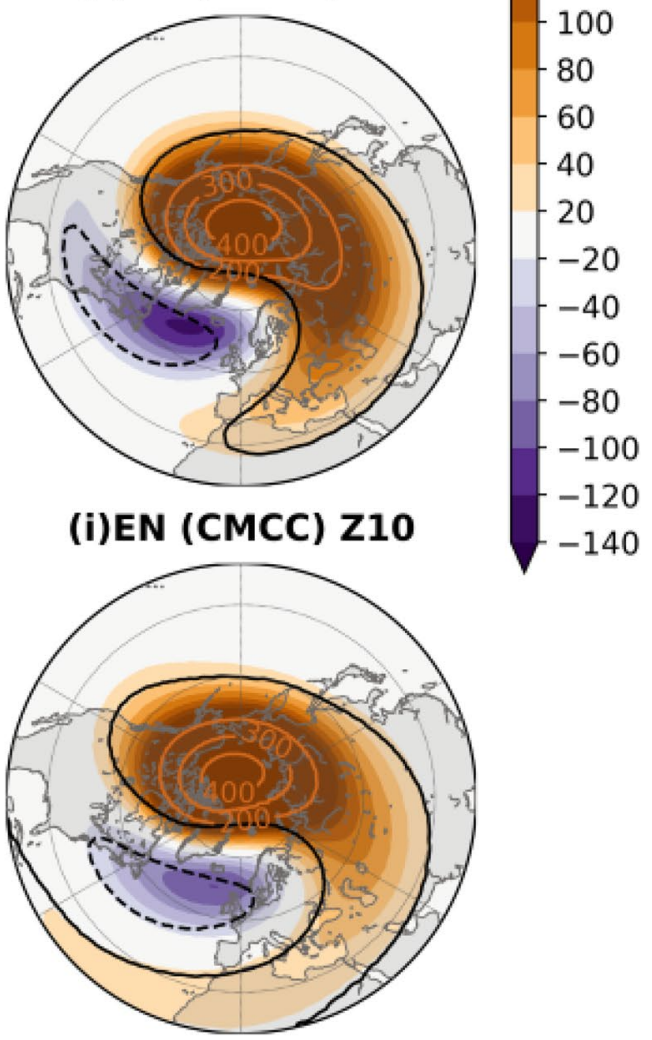

$\mathrm{m}$

140

120

100

80

60

20

20

$-40$

100

$-140$
Fig. 9 Ensemble-mean geopotential height anomalies for EN with respect to CTL in JFM at 1 (top), 5 (middle) and $10 \mathrm{hPa}$ (bottom): EC-EARTH (left), CNRM (middle), CMCC (right). Orange and magenta contours show values exceeding the color scale limit at $\pm 200, \pm 300, \pm 400$ and $\pm 500 \mathrm{~m}$. Black contours (solid for positive, dashed for negative anomalies) indicate statistically significant areas at the $95 \%$ confidence level fact present with a similar WN1 pattern in the middle-upper stratosphere (not shown; in agreement with Sassi et al. 2004; Manzini et al. 2006).

\subsection{Interpreting the polar cap temperature anomalies}

The presented results suggest that the ENSO circulation anomalies in the stratosphere are mainly associated with a propagating wave rather than a dissipating wave, in agreement with previous studies (e.g. Sassi et al. 2004; Manzini et al. 2006). It relies on the SST-forced, stationary tropospheric Rossby wave train, which propagates upwards and is eventually filtered into a WN1 pattern with no apparent wave breaking. As such, and since the fundamental mechanism underlying Rossby wave propagation is that potential vorticity of the fluid parcels is conserved (e.g. Vallis 2017), the ENSO temperature anomalies in the lower stratosphere, 
(a)LN (EC-EARTH) Z1

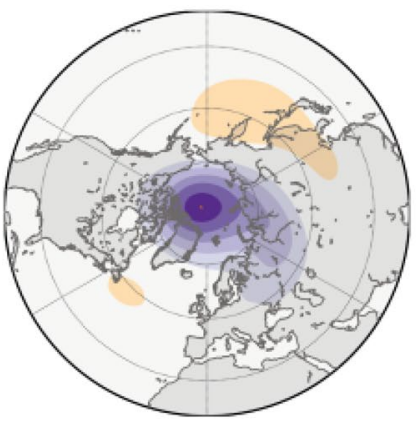

(d)LN (EC-EARTH) Z5

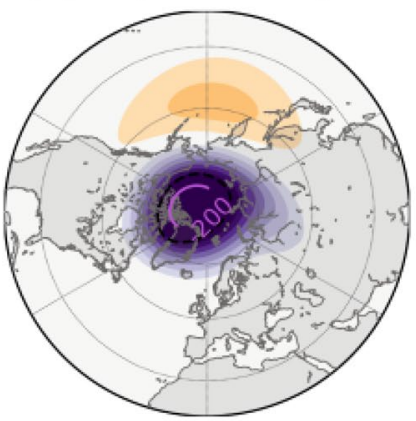

(g)LN (EC-EARTH) Z10

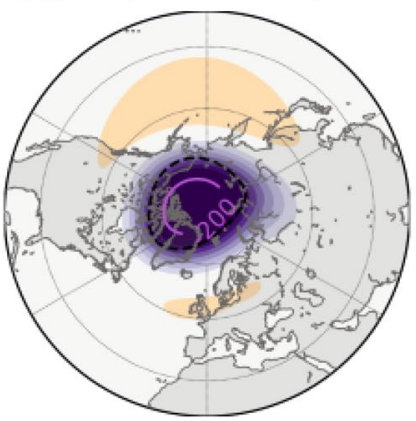

(b)LN (CNRM) Z1

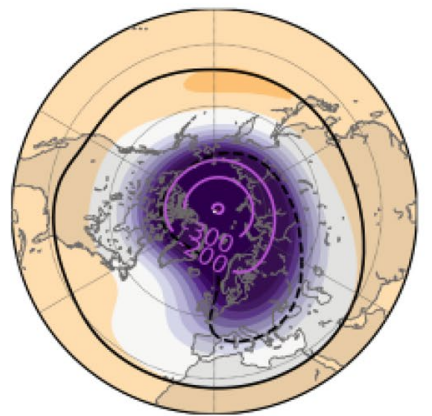

(e)LN (CNRM) Z5

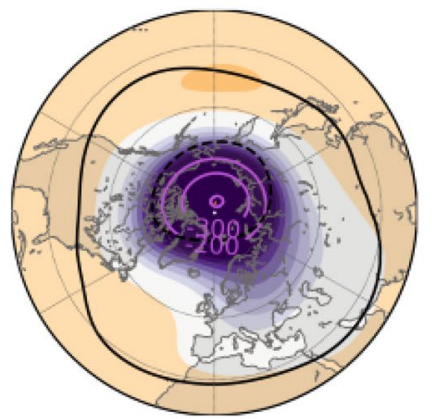

(h)LN (CNRM) Z10

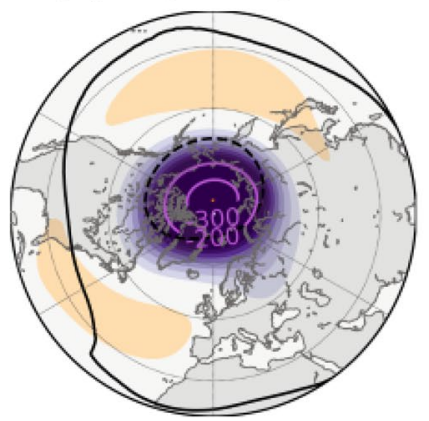

(c) LN (CMCC) Z1

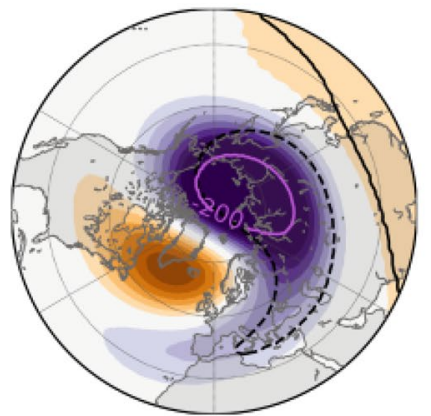

m 140

(f)LN (CMCC) Z5
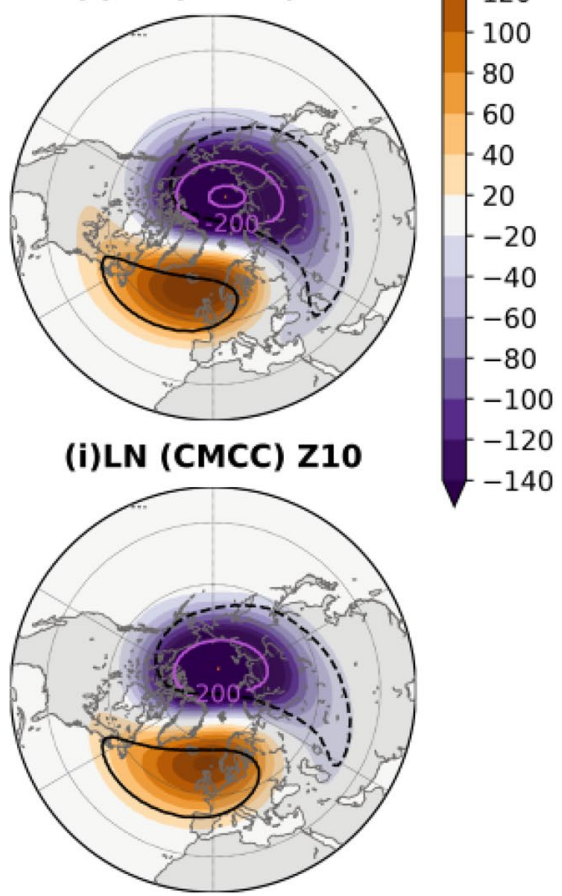

Fig. 10 Ensemble-mean geopotential height anomalies for LN with respect to CTL in JFM at 1 (top), 5 (middle) and $10 \mathrm{hPa}$ (bottom): EC-EARTH (left), CNRM (middle), CMCC (right). Orange and magenta contours show values exceeding the color scale limit at $\pm 200, \pm 300, \pm 400$ and $\pm 500 \mathrm{~m}$. Black contours (solid for positive, dashed for negative anomalies) indicate statistically significant areas at the $95 \%$ confidence level

the anomalous cyclonic circulation at high latitudes would be associated with anomalous stretching and differential cooling, to balance the increase in relative vorticity. In this case, however, the arching pathway of the wave train is such that it consistently spreads over the polar vortex region in the three models (Fig. 8), yielding a robust cooling of the polar cap (Figs. 3j-1, 6).

This framework of thermodynamic adjustment in the lower stratosphere, based on the balance of relative vorticity and stretching vorticity, operates at monthly time-scale in both the EN and LN experiments (see Figs. S3-S8 in the Supplementary Material). Further research is warranted to explore this hypothesis more in depth. 


\section{Summary and discussion}

We have used three state-of-the-art atmospheric models (EC-EARTH, CNRM and CMCC) and 50-member ensembles to study the impacts of idealized and symmetric El Niño and La Niña SST forcings on the Northern Hemisphere polar stratosphere in late winter (JFM). We have analyzed the ensemble-mean responses to strong ENSO events and found that they are similar to each other and to observations for La Niña, but not for El Niño, whose response is misrepresented by one of the models, and have profited from this difference to investigate the driving mechanisms of the ENSO teleconnection to the polar stratosphere. It is commonly considered that anomalous vertical propagation and breaking of planetary waves is key for the ENSO impact on the polar vortex. However, our results suggest that the ENSO response in the stratosphere is mainly driven by the upward propagation of the wavenumber-1 (WN1) component of the tropospheric Rossby wave train triggered by ENSO, starting over Canada at the tropopause and reaching the stratopause while tilting westward with height and projecting on the stratospheric Aleutian High. At lower stratospheric levels, the ENSOforced geopotential height anomalies at high latitudes are hydrostatically consistent with temperature anomalies around the polar vortex, which is interpreted in terms of shrinking/stretching since they are linked to a (reversible) distortion of the vortex without wave breaking. In accordance with quasi-geostrophic balance, the geopotential height anomalies associated with the upward propagation of the high-latitude WN1 pattern are responsible for the zonal wind anomalies related to the polar vortex. These temperature and zonal wind perturbations in the zonal average are those required to maintain thermal wind balance, implying a consistent anomalous vertical zonal-wind shear at middle-upper stratospheric levels. In the following, our main findings are discussed into context:

1. The two models that properly capture the well-known temperature and zonal wind El Niño signal in the latewinter stratosphere, i.e. warming at lower levels at polar latitudes and weakening of the polar vortex, indicate that the response to La Niña is symmetric in terms of opposite signed-patterns, but has smaller amplitude (about half). Mixed results are present in the literature concerning this (a)symmetry. The studies around the time of Brönnimann's (2007) review and in the subsequent years only addressed the impact of El Niño (e.g. Bell et al. 2009; Cagnazzo and Manzini 2009; Ineson and Scaife 2009) or mainly followed a linear El Niño-La Niña approach (e.g. Sassi et al. 2004; Taguchi and Hartmann 2006), although in some cases weaker and less significant anomalies for La Niña were reported (e.g. Manzini et al. 2006). More recently, Hurwitz et al. (2014), Rao and Ren (2016a, b) and Hardiman et al. (2019) found a large degree of symmetry in the polar stratospheric response to strong, canonical El Niño and La Niña events, as did Calvo et al. 2017 (El Niño) and Iza et al. 2016 (La Niña), using coupled simulations and several reanalysis products. Weinberger et al. (2019) examined a 41-member ensemble forced with observed SSTs and also found linearity for El Niño and La Niña. Trascasa-Castro et al. (2019) used an idealized, symmetric ENSO-like SST forcing of increasing amplitude: for strong events, they found winter anomalies in the zonal-mean zonal wind and EP flux/ divergence symmetric in sign but weaker in La Niña. Our results in the stratosphere are similar to what Mezzina et al. (2021) found for the troposphere, where the asymmetry in the magnitude of the response was related to the different energy available from the tropical Pacific in the two ENSO phases (linked to total SSTs). They suggested that the asymmetry in the tropospheric and surface response is not indicative of different mechanisms at play for the teleconnection of El Niño and La Niña, and here we reach the same conclusion for the impacts on the stratosphere.

2. The anomalous circulation over Canada of tropospheric origin is key for the ENSO response in both the lower and middle-upper stratosphere. In the case of El Niño (La Niña), it is an anticyclonic (cyclonic) circulation belonging to the large-scale Rossby wave train that constitutes the main feature of the tropospheric response to ENSO. In the two models that are properly capturing the stratospheric response to El Niño, this center of action extends north of $70^{\circ} \mathrm{N}$ even at $200 \mathrm{hPa}$ and increases with height in amplitude and extent, reinforcing the climatological Aleutian High in the middle-upper stratosphere. In the model that does not capture the weakening of the vortex and the lower-stratospheric warming, the anomalous center of action remains confined outside the polar cap at all levels, growing with height only in the upper stratosphere (above $10 \mathrm{hPa}$ ), where it also reinforces the Aleutian High. For La Niña, instead, the anomalous cyclonic circulation is correctly placed in all the models, possibly because of the slightly different arching path of the tropospheric Rossby wave train (Mezzina et al. 2021). As reviewed by Brönnimann (2007), the relevance of the ENSO-related geopotential height anomaly over Canada was already reported in the earlier works addressing the ENSO impacts on the stratosphere (van Loon and Labitzke 1987; Hamilton 1993a, b, 1995; Baldwin and O'Sullivan 1995), and was later stressed by other studies such as Sassi et al. (2004), Manzini et al. (2006) and Cagnazzo and Manzini (2009). In this latter work, the authors presented results from a "high-top" model that simulates, for El Niño, an anomalous anticyclone at $50 \mathrm{hPa}$ expanding over the polar cap, and from a "low-top" model for 
which the anomaly remains confined over Canada, similarly to EC-EARTH (see their Fig. 3); they also found that the zonal-mean temperature anomaly at $80^{\circ} \mathrm{N}$ was weaker in the "low-top" model (see their Fig. 1). That this anomalous circulation over Canada could play such a key role is somewhat in contrast with the more recent interpretation of the ENSO teleconnection to the stratosphere in terms of linear constructive (destructive) interference of the strengthened (weakened) tropospheric Aleutian Low during El Niño (La Niña) with the climatological wave pattern in the North Pacific mid-latitudes (e.g. Garfinkel and Hartmann 2008; Ineson and Scaife 2009; see the review by Domeisen et al. 2019). Our results instead agree with the early studies and stress the crucial role of the anomalous circulation over Canada.

3. We suggest that the temperature anomalies in the lower stratosphere could be interpreted in terms of shrinking/ stretching. The anomalous relative vorticity associated with the center of action over Canada, anticyclonic (cyclonic) for El Niño (La Niña), is balanced by anomalous shrinking (stretching) at polar latitudes, whose thermodynamical adjustment imply anomalous differential warming (cooling). We note that this anomalous circulation over Canada constitutes the key center of action of the wavenumber-1 (WN1) pattern that is filtered as it propagates vertically through the stratosphere. Our results suggest an alternative view to the current understanding of the ENSO impact on the stratosphere, based on the wavenumber-0 (WN0) response of the polar vortex driven by increased (decreased) wave forcing during El Niño (La Niña), associated with a deceleration (acceleration) and a warming (cooling) (see Domeisen et al. 2019 for a review). Our findings, instead, agree with previous works showing that the zonally-asymmetric, $\mathrm{WN} 1$ response to ENSO prevails over WNO, zonal-mean changes of the stratospheric circulation (e.g. Hamilton 1993a, 1993b; Baldwin and O'Sullivan 1995; Sassi et al. 2004), as well as with theoretical considerations where stationary, conservative, linear planetary waves, such as the ENSO-forced Rossby wave train, are incapable of inducing changes in the zonal-mean stratospheric flow (see Andrews et al. 1987 for a review).

4. The ENSO signal in the middle-upper stratosphere (above $50 \mathrm{hPa}$ ) projects onto a WN1 pattern, reminiscent of the climatological stationary eddy, for both El Niño and, more weakly, La Niña. The El Niño- (La Niña-) related geopotential height anomalies are in phase (out of phase) with the climatological wave pattern and reinforce (lessen) the climatological Aleutian High in the middle-upper stratosphere, resulting in a weakening (strengthening) of the polar vortex and a displacement towards the North Atlantic (North Pacific) sector. These features have been reported in previous works, from earlier (e.g. van Loon and Labitzke 1987; Hamilton 1993a, b, 1995; Sassi et al. 2004; Manzini et al. 2006) to more recent ones (e.g. Rao and Ren 2016a, b), but only for El Niño. Moreover, the anomalies show a westward tilt with height, which indicates upward propagation of wave activity, as reported in the same earlier studies (e.g. Hamilton 1995; Sassi et al. 2004; Manzini et al. 2006). Our results suggest that this response is related to the upward propagation of the lower-stratospheric anomalies and that thus wave breaking is not associated with this response (see point 3.); instead, the wave activity continues to propagate upwards into the higher stratosphere and the mesosphere, aligned with Sassi et al. (2004).

The results presented here complement those of Mezzina et al. $(2020,2021)$ in suggesting that the ENSO signal over the NAE region, the "canonical" SLP dipole between mid and high latitudes, is mostly driven by the tropospheric pathway. Indeed, even in ECEARTH the canonical SLP dipole is present during El Niño-although weaker over high latitudes compared to the other models-despite the erroneous stratospheric response. However, we acknowledge that lower-stratospheric circulation anomalies may propagate to the surface and induce SLP anomalies at polar/high latitudes via, for example, the same potential vorticity adjustment described above, as suggested by Ambaum and Hoskins (2002). Experiments with a stratosphere nudged to climatology (versus a free stratosphere), as in Jiménez-Esteve and Domeisen (2019), would allow to further isolate the stratospheric and tropospheric contributions to the ENSO-NAE signal.

There are many scientific questions concerning the ENSO impact on the stratosphere and the teleconnection to the NAE sector that remain open, but one that is not often discussed concerns a secondary center of action in SLP that was noticed, but not discussed, in Mezzina et al. (2020, 2021). It appears over Europe in the geopotential height field in the troposphere and lower stratosphere (Figs. 7, 8), albeit not always clearly. It is not straightforward to associate this anomaly with tropospheric processes, such as a split of the main wave train crossing the North Pacific-American sector (García-Serrano et al. 2011) or a secondary wave train triggered from the tropical Atlantic (Toniazzo and Scaife 2006), or with stratospheric ones, e.g. linked to the displacement of the polar vortex at $50-10 \mathrm{hPa}$, nor is it clear its role in the teleconnection, for which investigation is required.

\section{Appendix 1: observational composites}

Figure 11 shows observational composites of El Niño (a-d) and La Niña (e-h) in JFM. We use the same dataset (ECMWF ERA-20CR; Poli et al. 2016), period (1900-2010) 
Fig. 11 a-d JFM composites of El Niño anomalies using data from ECMWF ERA-20CR (Poli et al. 2016) over 1900-2010: zonal-mean zonal wind (a) and temperature (c); $100-\mathrm{hPa}$ geopotential height (b) and temperature (d). $\mathbf{e}-\mathbf{h}$ Same, but for La Niña. Green contours in the zonal wind panels show its climatology (contour interval: $10 \mathrm{~ms}^{-1}$ ) and the green line in the zonal-mean temperature panels depicts the tropopause level, according to the WMO's definition (lowest level at which the lapse rate decreases to $2{ }^{\circ} \mathrm{C} /$ $\mathrm{km}$ or less). Black contours (solid for positive, dashed for negative anomalies) indicate statistically significant areas at the $95 \%$ confidence level (a) EN ERA-20CR JFM [U] $\mathrm{ms}^{-1}$

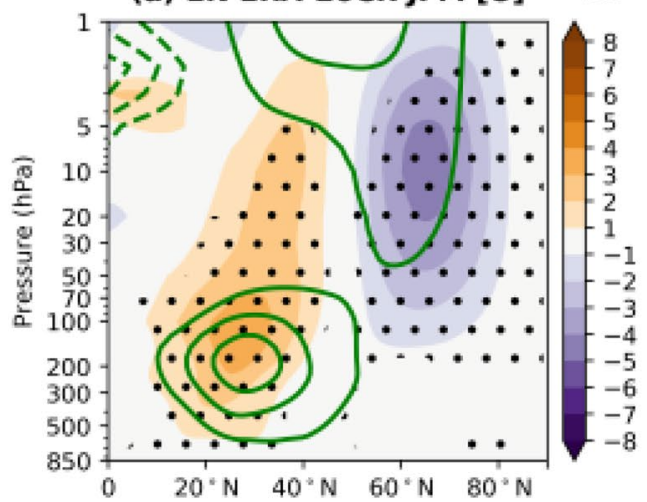

(c) EN ERA-20CR JFM [T]

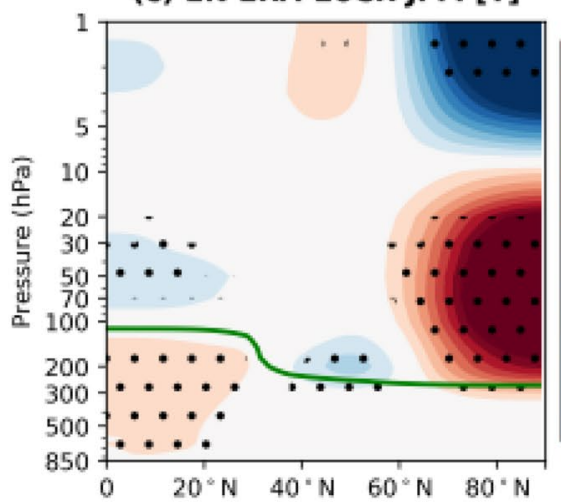

${ }^{\circ} \mathrm{C}$

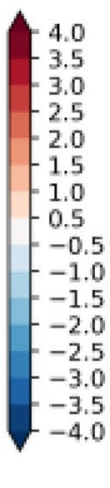

(e) LN ERA-20CR JFM [U] $\mathrm{ms}^{-1}$
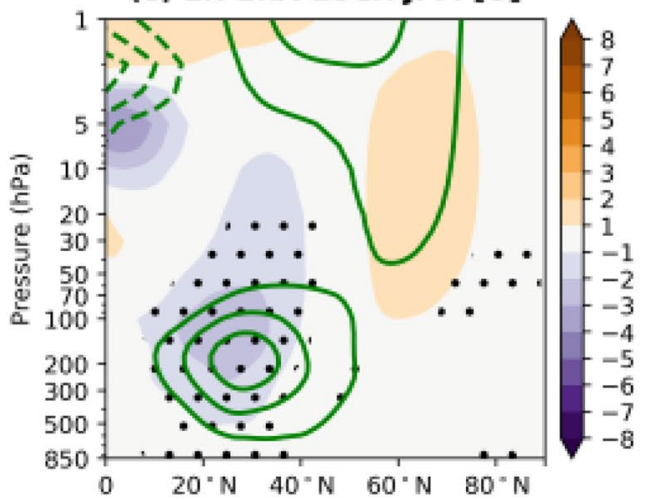

(g) LN ERA-20CR JFM [T]

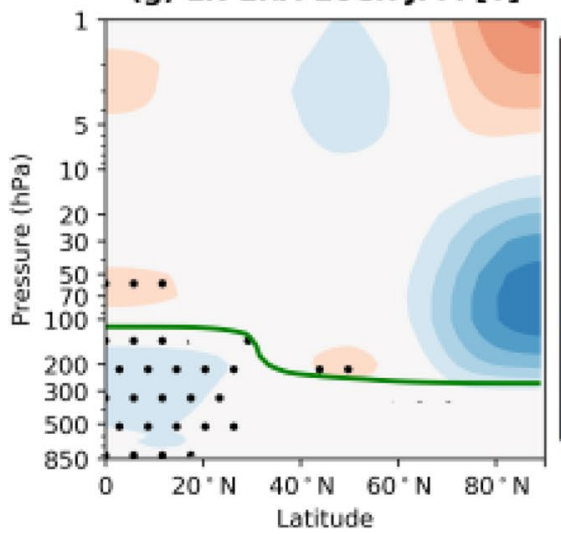

${ }^{\circ} \mathrm{C}$

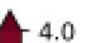

-4.0
-3.5
-3.0

-3.0
-2.5
-2.0

-2.0
-1.5
-1.5

$-1.0$

0.5
-0.5

$=-1.0$

-1.5
-2.0
-2.5

$-2.0$

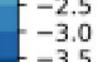

$-3.5$

$-4.0$

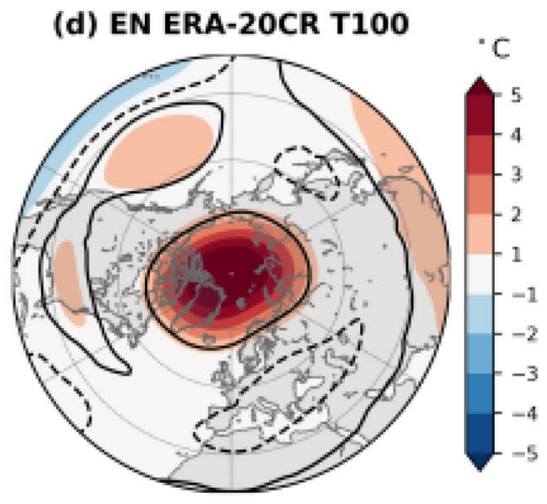

(f) LN ERA-20CR Z100

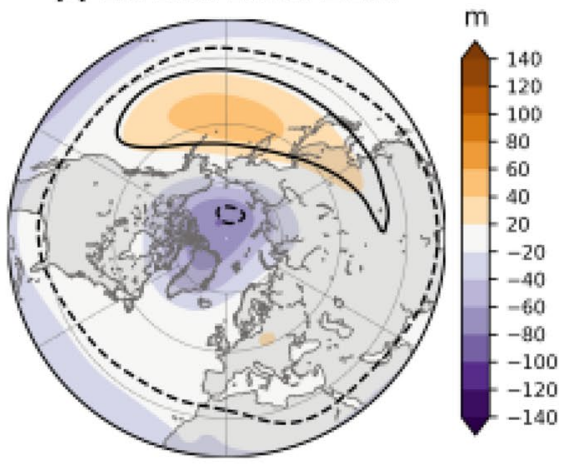

(h) LN ERA-20CR T100

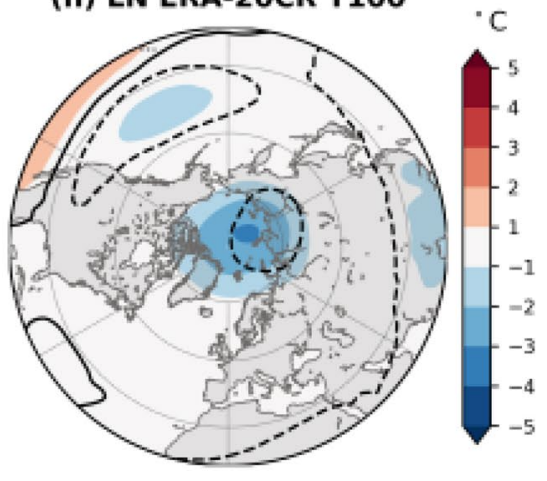


(a)EN(EC-EARTH) $v * T * 10$

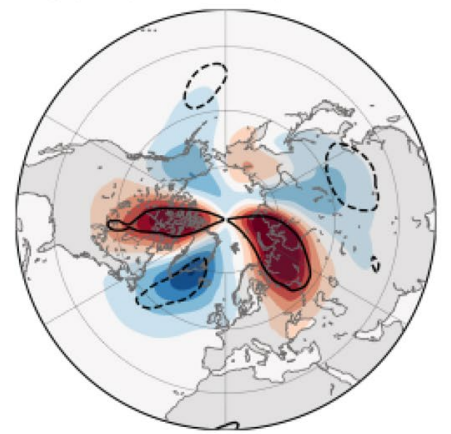

(d)EN(EC-EARTH) v*T*50

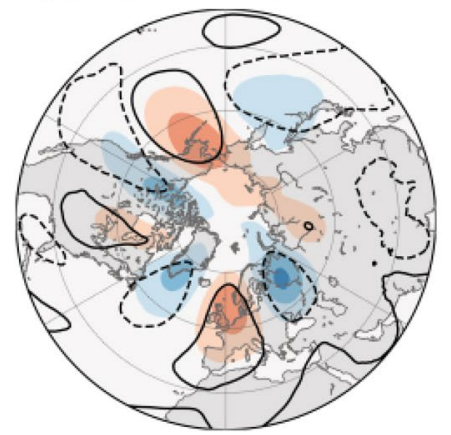

(b)EN(CNRM) v*T*10

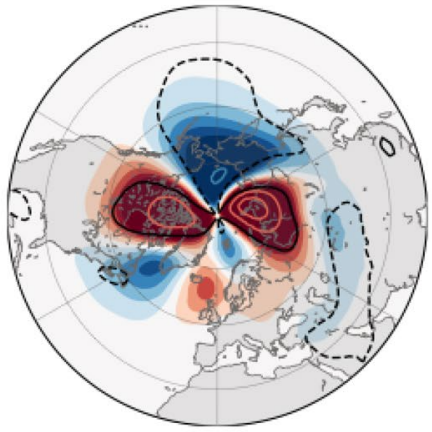

(e)EN(CNRM) v*T*50

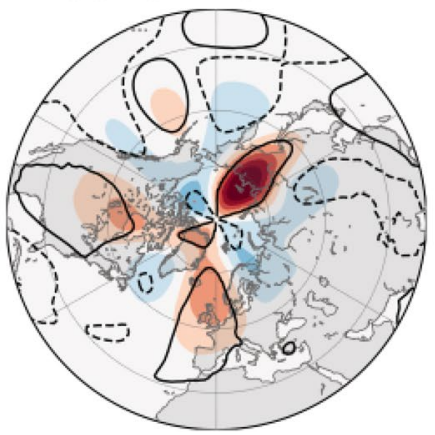

(c)EN(CMCC) $v * T * 10$

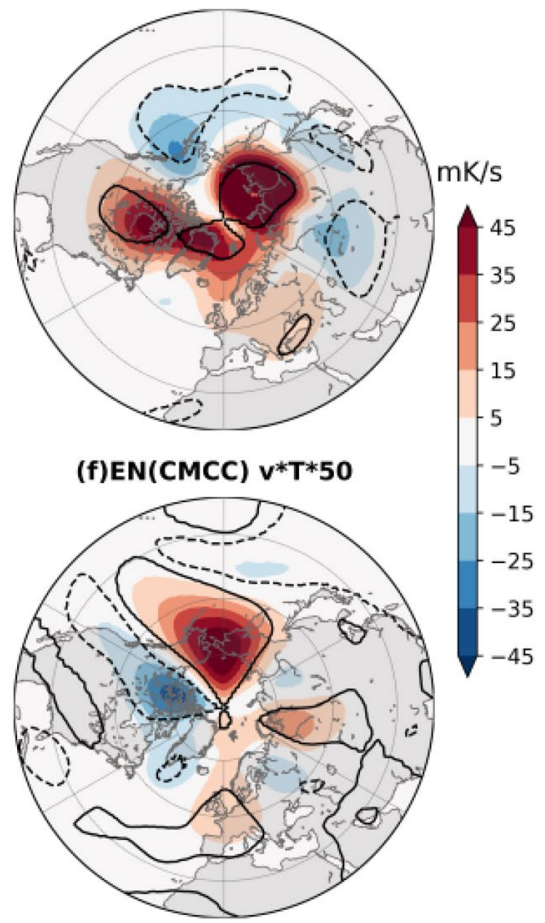

Fig. 12 Ensemble-mean eddy-heat flux anomalies for EN with respect to CTL in JF at 10 (top) and $50 \mathrm{hPa}$ (bottom): EC-EARTH (left), CNRM (middle), CMCC (right). Red and blue contours show values exceeding the color scale limit at $\pm 200, \pm 300, \pm 400$

and methods as in Mezzina et al. 2021, stratifying El Niño (La Niña) years according to a threshold of $+1(-1)$ standard deviation of the JFM Niño3.4-index. 18 EN and 19 LN years are selected, using SST from HadISST1.1.

\section{Appendix 2: longitude-latitude maps of $v^{*} T^{*}$}

Figure 12 shows maps of anomalous $v^{*} T^{*}$ in EN at 50 and $10 \mathrm{hPa}$, as discussed in Sect. 3.7.

Supplementary Information The online version contains supplementary material available at https://doi.org/10.1007/s00382-021-05836-3.

Acknowledgements This work was supported by the MEDSCOPE project. MEDSCOPE is part of ERA4CS, an ERA-NET initiated by JPI Climate, and funded by AEMET (ES), ANR (FR), BSC (ES), CMCC (IT), CNR (IT), IMR (BE) and Météo-France (FR), with co-funding by the European Union (Grant 690462). B.M. and J.G.-S. were supported by the "Contratos Predoctorales para la Formación de Doctores" (BES2016-076431) and "Ramón y Cajal" (RYC-2016-21181) programmes, respectively. F.M.P. was partially supported by the Spanish DANAE (CGL2015-68342-R) and GRAVITOCAST (ERC2018-092835) projects. Technical support at BSC (Computational Earth Sciences group) is sincerely acknowledged. We also thank the two anonymous reviewers for their helpful feedback. and $\pm 500 \mathrm{~m}$. Black contours (solid for positive, dashed for negative anomalies) indicate statistically significant areas at the $95 \%$ confidence level

Open Access This article is licensed under a Creative Commons Attribution 4.0 International License, which permits use, sharing, adaptation, distribution and reproduction in any medium or format, as long as you give appropriate credit to the original author(s) and the source, provide a link to the Creative Commons licence, and indicate if changes were made. The images or other third party material in this article are included in the article's Creative Commons licence, unless indicated otherwise in a credit line to the material. If material is not included in the article's Creative Commons licence and your intended use is not permitted by statutory regulation or exceeds the permitted use, you will need to obtain permission directly from the copyright holder. To view a copy of this licence, visit http://creativecommons.org/licenses/by/4.0/.

\section{References}

Ambaum MHP, Hoskins BJ (2002) The NAO troposphere-stratosphere connection. J Clim 15(14):1969-1978. https://doi.org/ 10.1175/1520-0442(2002)015\%3c1969:TNTSC\%3e2.0.CO;2

Andrews DG, Holton JR, Leovy CB (1987) Middle atmosphere dynamics. International geophysics series, vol 40. Academic Press, p 489

Ayarzagüena B, Ineson S, Dunstone NJ, Baldwin MP, Scaife AA (2018) Intraseasonal effects of El Niño-Southern oscillation on North Atlantic climate. J Clim 31:8861-8873. https://doi.org/10. 1175/JCLI-D-18-0097.1

Baldwin MP, O'Sullivan D (1995) Stratospheric effects of ENSOrelated tropospheric circulation anomalies. J Clim 8:649-667. 
https://doi.org/10.1175/1520-0442(1995)008\%3c0649:SEOERT\% 3e2.0.CO; 2

Bell CJ, Gray LJ, Charlton-Perez AJ, Joshi MM, Scaife AA (2009) Stratospheric communication of El Niño teleconnections to European winter. J Clim 22:4083-4096. https://doi.org/10.1175/2009J CLI2717.1

Benassi M, Conti G, Gualdi S et al (2021) El Niño teleconnection to the Euro-Mediterranean late-winter: the role of extratropical Pacific modulation. Clim Dyn. https://doi.org/10.1007/s00382$021-05768$-y (in press)

Brönnimann S (2007) Impact of El Niño-Southern Oscillation on European climate. Rev Geophys 45:RG3003. https://doi.org/10. 1029/2006RG000199

Butler AH, Polvani LM, Deser C (2014) Separating the stratospheric and tropospheric pathways of El Niño-Southern Oscillation teleconnections. Environ Res Let 9:024014. https://doi.org/10. 1088/1748-9326/9/2/024014

Cagnazzo C, Manzini E (2009) Impact of the stratosphere on the winter tropospheric teleconnections between ENSO and the North Atlantic and European Region. J Clim 22:1223-1238. https://doi.org/10.1175/2008JCLI2549.1

Calvo N, Garcia RR, Randel WJ, Marsh DR (2010) Dynamical mechanism for the increase in tropical upwelling in the lowermost tropical stratosphere during warm ENSO events. J Atmos Sci 67(7):2331-2340. https://doi.org/10.1175/2010JAS3433.1

Calvo N, Iza M, Hurwitz MM, Manzini E et al (2017) Northern hemisphere stratospheric pathway of different El Niño flavors in stratosphere-resolving CMIP5 models. J Clim 30(12):43514371. https://doi.org/10.1175/JCLI-D-16-0132.1

Charney JG, Drazin PG (1961) Propagation of planetary-scale disturbances from the lower into the upper atmosphere. J Geophys Res 66:83-109. https://doi.org/10.1029/JZ066i001p00083

Davini P, von Hardenberg J, Corti S et al (2017) Climate SPHINX: evaluating the impact of resolution and stochastic physics parameterisations in the EC-Earth global climate model. Geosci Model Dev 10:1383-1402. https://doi.org/10.5194/ gmd-10-1383-2017

Domeisen DIV, Butler AH, Fröhlich K et al (2015) Seasonal predictability over Europe arising from El Niño and stratospheric variability in the MPI-ESM Seasonal Prediction System. J Clim 28:256-271. https://doi.org/10.1175/JCLI-D-14-00207.1

Domeisen DIV, Garfinkel CI, Butler AH (2019) The teleconnection of El Niño Southern Oscillation to the stratosphere. Rev Geophys 57:5-47. https://doi.org/10.1029/2018RG000596

Free M, Seidel DJ (2009) Observed El Niño-Southern Oscillation temperature signal in the stratosphere. J Geophys Res 114:D23108. https://doi.org/10.1029/2009JD012420

García-Herrera R, Calvo N, Garcia RR, Giorgetta MA (2006) Propagation of ENSO temperature signals into the middle atmosphere: a comparison of two general circulation models and ERA-40 reanalysis data. J Geophys Res 111:D06101. https:// doi.org/10.1029/2005JD006061

García-Serrano J, Rodríguez-Fonseca B, Bladé I et al (2011) Rotational atmospheric circulation during North Atlantic-European winter: the influence of ENSO. Clim Dyn 37:1727-1743. https://doi.org/10.1007/s00382-010-0968-y

Garfinkel CI, Hartmann DL (2007) Effects of the El Niño-Southern Oscillation and the Quasi-Biennial Oscillation on polar temperatures in the stratosphere. J Geophys Res 112:D19112. https:// doi.org/10.1029/2007JD008481

Garfinkel CI, Hartmann DL (2008) Different ENSO teleconnections and their effects on the stratospheric polar vortex. J Geophys Res 113:D18114. https://doi.org/10.1029/2008JD009920

Hamilton K (1993a) A general circulation model simulation of El Nino effects in the extratropical northern hemisphere stratosphere. Geophys Res Let 20:1803-1806. https://doi.org/ 10.1029/93GL01782

Hamilton K (1993b) An examination of observed southern oscillation effects in the northern hemisphere stratosphere. J Atmos Sci 50:3468-3474. https://doi.org/10.1175/1520-0469(1993)050\% 3c3468:AEOOSO\%3e2.0.CO;2

Hamilton K (1995) Interannual variability in the northern hemisphere winter middle atmosphere in control and perturbed experiments with the GFDL SKYHI general circulation model. J Atmos Sci 52(1):44-66. https://doi.org/10.1175/1520-0469(1995)052\% 3c0044:IVITNH\%3e2.0.CO;2

Hardiman SC, Dunstone NJ, Scaife AA, Smith DM, Ineson S et al (2019) The impact of strong El Niño and La Niña events on the North Atlantic. Geophys Res Lett 46:2874-2883. https://doi.org/ 10.1029/2018GL081776

Harvey VL, Hitchman MH (1996) A climatology of the Aleutian High. J Atmos Sci 53:2088-2101. https://doi.org/10.1175/15200469(1996)053\%3c2088:ACOTAH\%3e2.0.CO;2

Harvey VL, Pierce RB, Hitchman MH (2002) A climatology of stratospheric polar vortices and anticyclones. J Geophys Res 107(D20):4442. https://doi.org/10.1029/2001JD001471

Horel JD, Wallace JM (1981) Planetary-scale atmospheric phenomena associated with the southern oscillation. Mon Weather Rev 109:813-829. https://doi.org/10.1175/1520-0493(1981)109\% 3c0813:PSAPAW\%3e2.0.CO;2

Hoskins BJ, Karoly DJ (1981) The steady linear response of a spherical atmosphere to thermal and orographic forcing. J Atmos Sci 38:1179-1196. https://doi.org/10.1175/1520-0469(1981)038\% 3c1179:TSLROA\%3e2.0.CO;2

Hurwitz MM, Calvo N, Garfinkel CI et al (2014) Extra-tropical atmospheric response to ENSO in the CMIP5 models. Clim Dyn 43:3367-3376. https://doi.org/10.1007/s00382-014-2110-z

Ineson S, Scaife AA (2009) The role of the stratosphere in the European climate response to El Niño. Nat Geosci 2(1):32-36. https:// doi.org/10.1038/ngeo381

Iza M, Calvo N, Manzini E (2016) The stratospheric pathway of La Niña. J Clim 29(24):8899-8914. https://doi.org/10.1175/ JCLI-D-16-0230.1

Jiménez-Esteve B, Domeisen DI (2019) Nonlinearity in the North Pacific atmospheric response to a linear ENSO forcing. Geophys Res Lett 46:2271-2281. https://doi.org/10.1029/2018GL081226

Kidston J, Scaife AA, Hardiman SC et al (2015) Stratospheric influence on tropospheric jet streams, storm tracks and surface weather. Nat Geosci 8:433-440. https://doi.org/10.1038/ngeo2424

King MP, Herceg-Bulić I, Bladé I et al (2018) Importance of late fall ENSO teleconnection in the Euro-Atlantic Sector. Bull Am Meteor Soc 99:1337-1343. https://doi.org/10.1175/BAMS-D-17-0020.1

Manzini E, Giorgetta MA, Esch M, Kornblueh L, Roeckner E (2006) The influence of sea surface temperatures on the northern winter stratosphere: ensemble simulations with the MAECHAM5 model. J Clim 19(16):3863-3881. https://doi.org/10.1175/JCLI3826.1

Matsuno T (1971) A dynamical model of the stratospheric sudden warming. J Atmos Sci 28:1479-1494. https://doi.org/10.1175/ 1520-0469(1971)028\%3c1479:ADMOTS\%3e2.0.CO;2

Mezzina B, García-Serrano J, Bladé I, Kucharski F (2020) Dynamics of the ENSO teleconnection and NAO variability in the North Atlantic-European Late Winter. J Clim 33:907-923. https://doi. org/10.1175/JCLI-D-19-0192.1

Mezzina B, Garcia-Serrano J, Bladé I et al (2021) Multi-model assessment of the late-winter ENSO teleconnection in the Euro-Atlantic sector. Clim Dyn. https://doi.org/10.1007/s00382-020-05415-y (in press)

Nigam S, DeWeaver E (2003) Stationary waves (orographic and thermally forced). In: Holton JR, Pyle JA, Curry JA (eds) Encyclopedia of atmospheric sciences. Academic/Elsevier Sci, London, pp 2121-2137 
Oehrlein J, Chiodo G, Polvani LM (2019) Separating and quantifying the distinct impacts of El Niño and sudden stratospheric warmings on North Atlantic and Eurasian wintertime climate. Atmos Sci Lett 20:e923. https://doi.org/10.1002/asl.923

Palmeiro FM, García-Serrano J, Bellprat O et al (2020) Boreal winter stratospheric variability in EC-EARTH: High-Top versus Low-Top. Clim Dyn 54:3135-3150. https://doi.org/10.1007/ s00382-020-05162-0

Palmeiro FM, Garcia-Serrano J, Ruggieri P, Batté L, Gualdi S (2021a) Intraseasonal modulation of Sudden Stratospheric Warmings by ENSO. Clim Dyn (submitted)

Palmeiro FM, García-Serrano J, Rodrigo M, Abalos M, Christiansen B, Yang S (2021b) Boreal winter stratospheric climatology: CMIP6 version. Clim Dyn (submitted)

Plumb RA (1985) On the three-dimensional propagation of stationary waves. J Atmos Sci 42(3):217-229. https://doi.org/10.1175/15200469(1985)042〈0217:OTTDPO $\rangle 2.0 . C O ; 2$

Poli P, Hersbach H, Dee DP, Berrisford P, Simmons AJ et al (2016) ERA-20C: an atmospheric reanalysis of the twentieth century. J Clim 29:4083-4097. https://doi.org/10.1175/JCLI-D-15-0556.1

Polvani LM, Sun L, Butler AH, Richter JH, Deser C (2017) Distinguishing stratospheric sudden warmings from ENSO as key drivers of wintertime climate variability over the North Atlantic and Eurasia. J Clim 30:1959-1969. https://doi.org/10.1175/ JCLI-D-16-0277.1

Rao J, Ren R (2016a) Asymmetry and nonlinearity of the influence of ENSO on the northern winter stratosphere: 1. Observ J Geophys Res Atmos 121:9000-9016. https://doi.org/10.1002/2015JD0245 20

Rao J, Ren R (2016b) Asymmetry and nonlinearity of the influence of ENSO on the northern winter stratosphere: 2. Model Study with WACCM. J Geophys Res Atmos 121:9017-9032. https://doi.org/ 10.1002/2015JD024521

Richter JH, Deser C, Sun L (2015) Effects of stratospheric variability on El Niño teleconnections. Environ Res Let 10:124021. https:// doi.org/10.1088/1748-9326/10/12/124021

Roehrig R, Beau I, Saint-Martin D et al (2020) The CNRM global atmosphere model ARPEGE-Climat 6.3: description and evaluation. J Adv Model Earth Syst 12:e2020MS002075. https://doi. org/10.1029/2020MS002075

Sanna A, Borrelli A, Athanasiadis P et al (2017) CMCC-SPS3: the CMCC seasonal prediction system 3. CMCC Tech Rep RP 0285:61
Sassi F, Kinnison D, Boville BA, Garcia RR, Roble R (2004) Effect of El Niño-Southern Oscillation on the dynamical, thermal, and chemical structure of the middle atmosphere. J Geophys Res 109:D17108. https://doi.org/10.1029/2003JD004434

Shaw TA, Perlwitz J, Weiner O (2014) Troposphere-stratosphere coupling: Links to North Atlantic weather and climate, including their representation in CMIP5 models. J Geophys Res Atmos 119:5864-5880. https://doi.org/10.1002/2013JD021191

Taguchi M, Hartmann DL (2006) Increased occurrence of stratospheric sudden warmings during El Niño simulated by WACCM. J Clim 19:324-332. https://doi.org/10.1175/JCLI3655.1

Titchner HA, Rayner NA (2014) The Met Office Hadley Centre sea ice and sea surface temperature data set, version 2: 1 . Sea ice concentrations. J Geophys Res Atmos 119:2864-2889. https:// doi.org/10.1002/2013JD020316

Toniazzo T, Scaife AA (2006) The influence of ENSO on winter North Atlantic climate. Geophys Res Lett 33:L24704. https://doi.org/10. 1029/2006GL027881

Trascasa-Castro P, Maycock AC, Scott Yiu YY, Fletcher JK (2019) On the linearity of the stratospheric and Euro-Atlantic sector response to ENSO. J Clim 32:6607-6626. https://doi.org/10.1175/ JCLI-D-18-0746.1

Vallis GK (2017) Atmospheric and oceanic fluid dynamics: fundamentals and large-scale circulation, 2nd edn. Cambridge University Press, p 946

Van Loon H, Labitzke K (1987) The Southern oscillation. Part V: the anomalies in the lower stratosphere of the northern hemisphere in winter and a comparison with the Quasi-Biennial Oscillation. Mon Weather Rev 115(2):357-369

Voldoire A, Saint-Martin D, Sénési S et al (2019) Evaluation of CMIP6 DECK experiments with CNRM- CM6-1. J Adv Model Earth Syst 11:2177-2213. https://doi.org/10.1029/2019MS001683

Waugh D, Polvani LM (2010) Stratospheric polar vortices. In the stratosphere, dynamics, transport and chemistry. Geophys Monogr Ser 190:43-57

Weinberger I, Garfinkel CI, White IP, Oman LD (2019) The salience of nonlinearities in the boreal winter response to ENSO: Arctic stratosphere and Europe. Clim Dyn 53:4591-4610

Publisher's Note Springer Nature remains neutral with regard to jurisdictional claims in published maps and institutional affiliations. 